\title{
Transition Operator Approach to Seismic Full-Waveform Inversion in Arbitrary Anisotropic Elastic Media
}

\author{
Morten Jakobsen ${ }^{1, *}$, Ivan Pšenčík ${ }^{2}$, Einar Iversen ${ }^{1}$ and \\ Bjørn Ursin ${ }^{3}$ \\ ${ }^{1}$ Department of Earth Science, University of Bergen, Norway. \\ 2 Institute of Geophysics, Czech Academy of Sciences, Czech Republic. \\ ${ }^{3}$ Department of Geoscience and Petroleum, NTNU and F. University of Bahia, Brazil.
}

Received 6 August 2018; Accepted (in revised version) 18 March 2019

\begin{abstract}
We generalize the existing distorted Born iterative T-matrix (DBIT) method to seismic full-waveform inversion (FWI) based on the scalar wave equation, so that it can be used for seismic FWI in arbitrary anisotropic elastic media with variable mass densities and elastic stiffness tensors. The elastodynamic wave equation for an arbitrary anisotropic heterogeneous medium is represented by an integral equation of the Lippmann-Schwinger type, with a 9-dimensional wave state (displacement-strain) vector. We solve this higher-dimensional Lippmann-Schwinger equation using a transition operator formalism used in quantum scattering theory. This allows for domain decomposition and novel variational estimates. The tensorial nonlinear inverse scattering problem is solved iteratively by using an expression for the Fréchet derivatives of the scattered wavefield with respect to elastic stiffness tensor fields in terms of modified Green's functions and wave state vectors that are updated after each iteration. Since the generalized DBIT method is consistent with the Gauss-Newton method, it incorporates approximate Hessian information that is essential for the reduction of multi-parameter cross-talk effects. The DBIT method is implemented efficiently using a variant of the Levenberg-Marquard method, with adaptive selection of the regularization parameter after each iteration. In a series of numerical experiments based on synthetic waveform data for transversely isotropic media with vertical symmetry axes, we obtained a very good match between the true and inverted models when using the traditional Voigt parameterization. This suggests that the effects of crosstalk can be sufficiently reduced by the incorporation of Hessian information and the use of suitable regularization methods. Since the generalized DBIT method for FWI in anisotropic elastic media is naturally target-oriented, it may be particularly suitable for applications to seismic reservoir characterization and monitoring. However, the theory and method presented here is general.
\end{abstract}

AMS subject classifications: 81U40, 74J25, 74J20, 45Dxx

*Corresponding author. Email addresses: morten.jakobsen@uib.no (M. Jakobsen), ip@ig.cas.cz (I. Pšenčík), Einar. Iversen@uib.no (E. Iversen), Bjorn. Ursin@ntnu.no (B. Ursin) 
Key words: Waveform inversion, time-lapse seismic, seismic inverse scattering, target-oriented inversion, Green's function.

\section{Introduction}

Seismic full-waveform inversion (FWI) promises images of the underground that are sharper and of higher resolution than those in conventional migration velocity analysis and travel time tomography [1-5]. However, FWI is characterized by several challenges [4], including a very strong sensitivity of the inversion to the initial model and a very high computational cost. The sensitivity of the FWI result to the starting model may be reduced by the use of multi-scale regularization methods [6,7], the calculation of higher-order Fréchet derivatives [8], envelope inversion [9] or some kind of renormalization $[10,11]$. Also, the computational cost may be significantly reduced using a simultaneous source method [12] and/or some kind of domain decomposition [13,14].

Many FWI methods have been developed on the basis of the acoustic wave equation [1-19]. The acoustic approximation may be adequate for many imaging purposes. For applications of the FWI method to seismic reservoir characterization and monitoring, however, it is essential to use the elastodynamic wave equation [20-25]. This is because shear-waves contain important information about the nature and content of pores and cracks $[22,26,27]$. To increase the realism of FWI, it may also be important to account for the effects of seismic anisotropy [28-44].

When a seismic wave propagates in a heterogeneous medium the wavelength is often large compared to the scale-size of pores, cracks and other heterogeneities so that the wave cannot 'see the details of the microstructure but only a smeared-out or averaged structure. If the heterogeneous microstructure has certain preferred directions then the average response on the macroscopic level will be anisotropic [26]. Many reservoirs are transversely isotropic due to fine layering [45] and/or the presence of aligned fractures [46]. Also, the shale layers that typically surround the reservoir formations are known to be transversely isotropic due to the presence of aligned clay minerals $[47,48]$. The CurieNeumann principle suggests that if the microstructure has certain symmetries then the macroscopic response will show the same symmetries [27,49]. However, it is important to keep in mind that symmetries in geology are always approximate [49]. In any case, seismic anisotropy is an academically interesting topic of great practical interest.

The goal of FWI in anisotropic elastic media is to reconstruct multiple parameter fields from the observed waveforms. In a general medium, there are 21 independent elastic stiffness parameters and the mass density [27]. The number of independent parameters can, of course, be reduced when material symmetries are present [27], but multiparameter FWI can be very challenging even for just a few independent parameters [36]. Involving multiple parameters increases the nonlinearity of the inversion process and also introduces parameter crosstalk; that is, the influence of one elastic parameter on the data associated with another elastic parameter $[32,39,50]$. 
The problem with parameter crosstalk is particularly significant when using a gradientbased steepest-descent method [39]. This is because the steepest-descent method does not account for the multiparameter character of the anisotropic elastic FWI problem [39]. To mitigate the problem with parameter crosstalk, one can try to change the parameterization of seismic anisotropy [33-35,37,40-43,51] and/or to include Hessian information in the inversion process [39]. However, it is not obvious that a parameterization of seismic anisotropy is optimal for FWI in heterogeneous anisotropic elastic media if the socalled radiation patterns discussed in [33-35,37,40-43,51] are not overlapping very much. This is because such radiation patterns are calculated under the unrealistic simplifying assumption of homogeneity and isotropy. In this paper, we derive expressions for the Fréchet derivatives of the scattered wavefield with respect to perturbations of different stiffness tensor elements in terms of modified Green's functions and wavefields that are updated after each iteration during the nonlinear inversion process. These expressions represent a more realistic model for the response of a localized stiffness perturbation within a heterogeneous anisotropic elastic medium, because nonlinear effects of multiple scattering are accounted for. The study of [39] implies that it is equally or more important to account for Hessian information in the iterative inversion process, than to search for the optimal parameterization of the model, when performing multi-parameter FWI in anisotropic elastic media. Our experience is that one can often obtain very good inversion results on the basis of the Voigt stiffness parameterization, provided that Hessian information is properly accounted for and a suitable regularization method is used to deal with the ill-posed nature of the multi-parameter FWI problem. In any case, many researchers have already studied the benefits of using different parameterizations of seismic anisotropy within the context of FWI, and there seems to be a need for more investigations of the importance of Hessian information in this context. Therefore, in this study we develop a Gauss-Newton consistent scattering approach to FWI in anisotropic elastic media that takes into account such Hessian information in an approximate manner. More specifically, we shall develop an elastodynamic generalization of the distorted Born iterative T-matrix (DBIT) method that Jakobsen and Ursin [5] introduced for acoustic FWI. Since our generalization of the DBIT algorithm involves not only elastodynamic effects and seismic anisotropy but is also naturally target-oriented [52], it may be particularly suitable for applications to seismic reservoir characterization and monitoring.

The outline of this paper can be described as follows. In "The nonlinear direct scattering problem", we discuss seismic forward modeling in arbitrary anisotropic elastic media. First we show that the particle displacement and the strain fields satisfies two coupled integral equations. Then we express these two coupled integral equations in operator notation and combine them into a single integral equation of the LippmannSchwinger type, which can be solved using an integral operator formalism known from the scalar case $[5,10,14,48,53-56]$. In "The nonlinear inverse scattering problem", we present an anisotropic elastic version of the DBIT method $[5,14]$; and also provide some details for implementation. In "Numerical examples", we test it's performance on synthetic seismic waveform data". The new elastodynamic DBIT algorithm can in principle 
be used with any parameterization of seismic anisotropy, but we show numerical results obtained using the Voigt stiffness parameterization only. In "Concluding remarks", we also present some ideas for future work. The paper also contains three appendices with modified Green's functions, a derivation and a summary of the DBIT algorithm.

\section{The nonlinear direct scattering problem}

\subsection{Two coupled integral equations}

We work in the frequency domain and assume that the components of the particle displacement vector $u_{i}(\mathbf{x})$ at point $\mathbf{x}$ due to a single-force source density with components $S_{i}(\mathbf{x})$ in an anisotropic elastic medium with stiffness tensor component field $C_{i j k l}(\mathbf{x})$ and mass density $\rho(\mathbf{x})$ satisfy the elastodynamic wave equation $[57,58]$

$$
\left[C_{i j k l}(\mathbf{x}) u_{k, l}(\mathbf{x})\right]_{, j}+\rho(\mathbf{x}) \omega^{2} u_{i}(\mathbf{x})=-S_{i}(\mathbf{x}) .
$$

We assume that $u_{i}(\mathbf{x})$ is proportional to $e^{i \omega t}$, where $\omega$ is the angular frequency. Under the assumption that the medium is unbounded and the wavefield goes to zero at infinity, the solution of the elastodynamic wave equation (2.1) is given by [57]

$$
u_{i}(\mathbf{x})=\int d \mathbf{x}^{\prime} g_{i j}\left(\mathbf{x}, \mathbf{x}^{\prime}\right) S_{j}\left(\mathbf{x}^{\prime}\right),
$$

where the Green's function $g_{i j}\left(\mathbf{x}, \mathbf{x}^{\prime}\right)$ per definition satisfies the following equation:

$$
\left[C_{i j k l}(\mathbf{x}) g_{k m, l}\left(\mathbf{x}, \mathbf{x}^{\prime}\right)\right]_{, j}+\rho(\mathbf{x}) \omega^{2} g_{i m}\left(\mathbf{x}, \mathbf{x}^{\prime}\right)=-\delta_{i m} \delta\left(\mathbf{x}-\mathbf{x}^{\prime}\right) .
$$

In order to derive a volume integral equation for the components $u_{i}(\mathbf{x})$ of the particle displacement field, we first decompose the stiffness tensor components $C_{i j k l}(\mathbf{x})$ and the corresponding mass density as $\rho(\mathbf{x})$ as

$$
\begin{aligned}
& C_{i j k l}(\mathbf{x})=C_{i j k l}^{(0)}(\mathbf{x})+\Delta C_{i j k l}(\mathbf{x}), \\
& \rho(\mathbf{x})=\rho^{(0)}(\mathbf{x})+\Delta \rho(\mathbf{x}),
\end{aligned}
$$

where $C_{i j k l}^{(0)}(\mathbf{x})$ and $\rho^{(0)}(\mathbf{x})$ are the stiffness elements and mass density of an arbitrary reference medium and $\Delta C_{i j k l}(\mathbf{x})$ and $\Delta \rho(\mathbf{x})$ are the corresponding perturbations, that are not necessarily small. From Eqs. (2.1), (2.4) and (2.5), we get

$$
\left[C_{i j k l}^{(0)}(\mathbf{x}) u_{k, l}(\mathbf{x})\right]_{, j}+\rho(\mathbf{x}) \omega^{2} u_{i}(\mathbf{x})=-S_{i}(\mathbf{x})-\left[\Delta C_{i j k l}(\mathbf{x}) u_{k, l}(\mathbf{x})\right]_{, j}-\omega^{2} \Delta \rho(\mathbf{x}) u_{i}(\mathbf{x}) .
$$

By treating the two last (contrast-source) terms on the right-hand side of Eq. (2.6) just like ordinary sources and using Eq. (2.2), we obtain

$$
u_{i}(\mathbf{x})=u_{i}^{(0)}(\mathbf{x})+\int d \mathbf{x}^{\prime} g_{i j}^{(0)}\left(\mathbf{x}, \mathbf{x}^{\prime}\right)\left\{\left[\Delta C_{j k l m}\left(\mathbf{x}^{\prime}\right) u_{l, m}\left(\mathbf{x}^{\prime}\right)\right]_{, k}+\omega^{2} \Delta \rho\left(\mathbf{x}^{\prime}\right) u_{i}\left(\mathbf{x}^{\prime}\right)\right\},
$$


where

$$
u_{i}^{(0)}(\mathbf{x})=\int d \mathbf{x}^{\prime} g_{i j}^{(0)}\left(\mathbf{x}, \mathbf{x}^{\prime}\right) S_{j}\left(\mathbf{x}^{\prime}\right),
$$

and $g_{i j}^{(0)}\left(\mathbf{x}, \mathbf{x}^{\prime}\right)$ is the solution of

$$
\left[C_{i j k l}^{(0)}(\mathbf{x}) g_{k m, l}^{[0]}\left(\mathbf{x}, \mathbf{x}^{\prime}\right)\right]_{, j}+\rho(\mathbf{x}) \omega^{2} g_{i m}^{(0)}\left(\mathbf{x}, \mathbf{x}^{\prime}\right)=-\delta_{i m} \delta\left(\mathbf{x}-\mathbf{x}^{\prime}\right) .
$$

In Eq. (2.7), $u_{i}^{(0)}(\mathbf{x}, \omega)$ is the wavefield in the reference model due to the vectorial source with components $S_{i}\left(\mathbf{x}^{\prime}\right)$, and $g_{i j}^{(0)}\left(\mathbf{x}, \mathbf{x}^{\prime}, \omega\right)$ is the reference medium Green's function. If the reference medium is homogeneous then one can use simple analytical formulae to calculate the Green's function for the reference medium, see Appendix A. Otherwise, the reference Green's functions must be computed using ray theory [58] or numerically [59].

The integral equation (2.7) is not convenient for inversion, since it contains the derivatives of the stiffness perturbation tensor. However, one can effectively move the gradient operator from the stiffness perturbation to the Green's function if one assumes that the wavefield approaches zero at infinity. By performing a partial integration [58] and making use of the well-known symmetries of the elastic stiffness tensor, the integral equation (2.7) can be rewritten exactly as

$$
u_{i}(\mathbf{x})=u_{i}^{(0)}(\mathbf{x})+\int d \mathbf{x}^{\prime} M_{i j k}^{(0)}\left(\mathbf{x}, \mathbf{x}^{\prime}\right) \Delta C_{j k l m}\left(\mathbf{x}^{\prime}\right) \epsilon_{l m}\left(\mathbf{x}^{\prime}\right)+\omega^{2} \int d \mathbf{x}^{\prime} g_{i j}^{(0)}\left(\mathbf{x}, \mathbf{x}^{\prime}\right) \Delta \rho\left(\mathbf{x}^{\prime}\right) u_{j}\left(\mathbf{x}^{\prime}\right) .
$$

In order to allow for the use of abbreviated subscripts for symmetric tensors (in the next section), we are introducing here the well-known strain tensor $\epsilon_{k l}(\mathbf{x})=$ $(1 / 2)\left[u_{k, l}(\mathbf{x})+u_{l, k}(\mathbf{x})\right]$ and a less well-known third-rank tensor $M^{(0)}\left(\mathbf{x}, \mathbf{x}^{\prime}\right)$, which has the following tensor components [48,60]:

$$
M_{i j k}^{(0)}\left(\mathbf{x}, \mathbf{x}^{\prime}\right)=-\frac{1}{2}\left[g_{i j, k^{\prime}}^{(0)}\left(\mathbf{x}, \mathbf{x}^{\prime}\right)+g_{i k, j^{\prime}}^{(0)}\left(\mathbf{x}, \mathbf{x}^{\prime}\right)\right] .
$$

By taking spatial derivatives of Eq. (2.10) at position $\mathbf{x}$, one can show that the strain field satisfies the following integral equation [60]:

$$
\epsilon_{i j}(\mathbf{x})=\epsilon_{i j}^{(0)}(\mathbf{x})+\int d \mathbf{x}^{\prime} \Gamma_{i j k l}^{(0)}\left(\mathbf{x}, \mathbf{x}^{\prime}\right) \Delta C_{j k l m}\left(\mathbf{x}^{\prime}\right) \epsilon_{l m}\left(\mathbf{x}^{\prime}\right)+\omega^{2} \int d \mathbf{x}^{\prime} M_{i j k}^{(0)}\left(\mathbf{x}, \mathbf{x}^{\prime}\right) \Delta \rho\left(\mathbf{x}^{\prime}\right) u_{k}\left(\mathbf{x}^{\prime}\right),
$$

where the components of the fourth-rank tensor $\Gamma^{(0)}\left(\mathbf{x}, \mathbf{x}^{\prime}\right)$ are defined by $[48,60]$

$$
\Gamma_{i j k l}^{(0)}\left(\mathbf{x}, \mathbf{x}^{\prime}\right)=\frac{1}{4}\left[g_{i j, k l^{\prime}}^{(0)}\left(\mathbf{x}, \mathbf{x}^{\prime}\right)+g_{k j, i l^{\prime}}^{(0)}\left(\mathbf{x}, \mathbf{x}^{\prime}\right)+g_{i l, k j^{\prime}}^{(0)}\left(\mathbf{x}, \mathbf{x}^{\prime}\right)+g_{k l, i j^{\prime}}^{(0)}\left(\mathbf{x}, \mathbf{x}^{\prime}\right)\right] .
$$

$\Gamma_{i j k l}^{(0)}\left(\mathbf{x}, \mathbf{x}^{\prime}\right)$ gives the $i j$ component of strain at $\mathbf{x}$ due to the $k l$ component of stress at $\mathbf{x}^{\prime}$.

In accordance with the terminology used in dynamic effective medium theory [60], one may refer to the third- and fourth-rank tensors $M^{(0)}$ and $\Gamma^{(0)}$ as modified Green's 
functions [61,62]. Explicit analytical expressions for the (modified) Green's functions of a homogeneous isotropic elastic medium are given in Appendix A. These expressions are used in conjunction with the integral operator formalism derived in the next section to compute (modified) Green's functions for more complicated heterogeneous anisotropic elastic media required for direct iterative nonlinear inversion $[5,14]$.

\subsection{Integral operator formalism}

To allow for a rigorous mathematical derivation based on concepts from modern physics, we now express the two coupled integral equations (2.10) and (2.12) in operator notation using Dirac's bracket notation for linear integral operators and state-vectors in dual Hilbert spaces [55] as

$$
\begin{aligned}
& |u\rangle=\left|u^{(0)}\right\rangle+M^{(0)} \Delta C|\epsilon\rangle+\omega^{2} g^{(0)} \Delta \rho|u\rangle, \\
& |\epsilon\rangle=\left|\epsilon^{(0)}\right\rangle+\omega^{2} M^{(0)} \Delta \rho|u\rangle+\Gamma^{(0)} \Delta C|\epsilon\rangle,
\end{aligned}
$$

where the matrix elements of the (local) scattering potential operators $\Delta C$ and $\Delta \rho$ are given by $\left\langle x|\Delta C| x^{\prime}\right\rangle=\Delta C_{i j k l}(\mathbf{x}) \delta\left(\mathbf{x}-\mathbf{x}^{\prime}\right)$ and $\left\langle x|\Delta \rho| x^{\prime}\right\rangle=\Delta \rho(\mathbf{x}) \delta\left(\mathbf{x}-\mathbf{x}^{\prime}\right)$ in the real-space coordinate representation.

Following [5,60], we now rewrite the two coupled integral equations (2.14)-(2.15) for the displacement and strain fields in block-matrix form as

$$
|\psi\rangle=\left|\psi^{(0)}\right\rangle+G^{(0)} V|\psi\rangle,
$$

where

$$
|\psi\rangle=\left[\begin{array}{c}
|u\rangle \\
|\epsilon\rangle
\end{array}\right], \quad\left|\psi^{(0)}\right\rangle=\left[\begin{array}{l}
\left|u^{(0)}\right\rangle \\
\left|\epsilon^{(0)}\right\rangle
\end{array}\right]
$$

represent the combined displacement-strain state in the actual and reference medium, respectively; and

$$
G^{(0)}=\left[\begin{array}{cc}
\omega^{2} g^{(0)} & M^{(0)} \\
\omega^{2} M^{(0)} & \Gamma^{(0)}
\end{array}\right], \quad V=\left[\begin{array}{cc}
\Delta \rho & 0 \\
0 & \Delta C
\end{array}\right]
$$

are $2 \times 2$ matrices of integral operators.

The higher-dimensional integral equation (2.16) for the combined displacement-strain state vector $|\psi\rangle$ has the same form as the Lippmann-Schwinger equation in quantum scattering theory $[55,56]$. This is an agreeable feature because it permits us to modify the highly developed perturbative and iterative methods developed to solve scattering problems in quantum mechanics for the use in seismic modelling. Following [55,56], we now introduce a transition operator (or T-matrix) by

$$
V|\psi\rangle=T\left|\psi^{(0)}\right\rangle,
$$


where $V$ is given in Eq. (2.18) and $T$ is the transition operator. We can then rewrite the Lippmann-Schwinger equation (2.16) as

$$
|\psi\rangle=\left|\psi^{(0)}\right\rangle+G^{(0)} T\left|\psi^{(0)}\right\rangle .
$$

In order to obtain an integral equation for the transition operator, we first apply the scattering potential operator $V$ on the integral equation (2.20) from the left

$$
V|\psi\rangle=V\left|\psi^{(0)}\right\rangle+V G^{(0)} T\left|\psi^{(0)}\right\rangle .
$$

By using the definition (2.19) of the T-matrix in conjunction with Eq. (2.21), we obtain

$$
T\left|\psi^{(0)}\right\rangle=V\left|\psi^{(0)}\right\rangle+V G^{(0)} T\left|\psi^{(0)}\right\rangle .
$$

Since the reference medium and hence the reference state vector $\left|\psi^{(0)}\right\rangle$ may be chosen arbitrarily, it follows that the transition operator also satisfies an integral equation of the Lippmann-Schwinger type [5]

$$
T=V+V G^{(0)} T,
$$

which has the following exact formal solution

$$
T=\left(I-V G^{(0)}\right)^{-1} V,
$$

where $I$ is the identity operator.

Since the above solution is equivalent to (a well-known expression $[55,56]$ derived in Appendix B):

$$
T=V\left(I-G^{(0)} V\right)^{-1},
$$

the transition operator also satisfies the following integral equation

$$
T=V+T G^{(0)} V \text {. }
$$

Therefore, it follows from Eqs. (2.20) and (2.26) that the integral equation (2.16) is equivalent to

$$
|\psi\rangle=\left|\psi^{(0)}\right\rangle+G V\left|\psi^{(0)}\right\rangle,
$$

where the Green's operator $G$ for the actual medium satisfies the following integral equation

$$
G=G^{(0)}+G^{(0)} T G^{(0)},
$$

that in conjunction with Eqs. (2.16) and (2.27) will be used later in the context of nonlinear inverse scattering to update the forward model and the data residuals after each iteration.

If Gaussian elimination is used to solve the integral equation (2.23) or (2.26) via matrix inversion in the real-space coordinate representation then the asymptotic computational complexity of calculating the T-matrix in the general anisotropic case will scale like $N^{3}$ for large numbers of grid blocks $N$. However, Gaussian elimination is not optimal [63] and so we may try to accelerate the T-matrix approach by domain decomposition [14]. Also, one can avoid the need to invert a huge matrix by using a variational approach discussed at the end of this section. 


\subsection{Domain decomposition via scattering-path operator formalism}

Without loss of generality, we assume that the arbitrary heterogeneous and anisotropic background medium contains a population of $N_{d}$ heterogeneous (and non-overlapping) domains, so that the scattering potential operator $V$ given in Eq. (2.18) for the whole model can be written in diagonal block matrix form as

$$
V=\left\{V_{\alpha} \delta_{\alpha \beta}\right\}, \quad \alpha, \beta=1, \cdots, N_{d},
$$

where $\delta_{\alpha \gamma}$ is the Kronecker delta and the operator $V_{\alpha}$ represents the scattering potential operator for the $\alpha$ th domain, that is generally anisotropic and heterogeneous. We can also write the Green's function operator $G^{(0)}$ in non-diagonal block matrix form as

$$
G^{(0)}=\left\{G_{\alpha \beta}^{(0)}\right\}, \quad \alpha, \beta=1, \cdots, N_{d},
$$

where $G_{\alpha \beta}^{(0)}$ is related to the interaction between two domains $\beta$ and $\alpha$ that may or may not the same; in the sense that the source and receiver may or may not be in the same domain. Following $[14,64]$, we then assume that the corresponding transition operator can be written as

$$
T=\left\{T_{\alpha \beta}\right\}, \quad \alpha, \beta=1, \cdots, N_{d},
$$

where the scattering-path operator (SPO) elements $T_{\alpha \beta}$ account for all scattering events that start and end in domains $\alpha$ and $\beta$, respectively [64]. By using the above decomposition in conjunction with the Lippmann-Schwinger equation (2.23) for the transition operator, one can show that [14]

$$
T_{\alpha \gamma}=V_{\alpha} \delta_{\alpha \gamma}+V_{\alpha} \sum_{\beta=1}^{N_{d}} G_{\alpha \beta}^{(0)} T_{\beta \gamma} .
$$

The above equation can be rewritten exactly as $[14,60]$

$$
T_{\alpha \gamma}=t_{\alpha} \delta_{\alpha \gamma}+t_{\alpha} \sum_{\beta=1}^{N_{d}} G_{\alpha \beta}^{(0)}\left(1-\delta_{\alpha \beta}\right) T_{\beta \gamma},
$$

where

$$
t_{\alpha}=V_{\alpha}+V_{\alpha} G_{\alpha \alpha}^{(0)} t_{\alpha}
$$

is the t-matrix for the $\alpha$ th domain; it is considered isolated from all the other domains (see also [48]). This scattering-path operator decomposition is highly suitable for implementation on a parallel computing platform. On the basis of the scalar wave equation, [14] discuss a block-matrix representation of the SPO and an interesting link to Strassen's [63] algorithm for fast matrix inversion that could be used to further develop and implement the above equations in the real-space coordinate representation. 


\subsection{Estimates of the transition operator via a variational approach}

In quantum scattering theory, researchers $[55,56]$ use two main types of approaches; namely, perturbation theory and variational methods. Perturbation theory is commonly used in the seismic community, but variational methods are less well-known. Perturbation theory is often based on the assumption of weak scattering (e.g., the distorted Born approximation). Renormalization methods can in principle be used to eliminate the weak scattering assumption, but this is easier to do for the nonlinear direct scattering problem than for the corresponding nonlinear inverse scattering problem [11]. Variational approaches are fundamentally different from perturbation theory, since they are not based on an expansion of the wavefield or the various integral operators in powers of a coupling constant. We here focus on the application of the variational approach to direct nonlinear scattering, but it is possible that variational methods may be useful in future attempts to reduce the sensitivity of scattering-based FWI on the starting model.

The purpose of variational formulas is to develop approximations for the wavefield, the Green's function or the T-matrix [56]. In this section, we shall modify the variational formulas that Newton [56] derived for the estimation of the transition operator in quantum scattering theory so that it can be used to estimate the transition operator in elastodynamic scattering theory. Let us first insert Eq. (2.23) into Eq. (2.26) to obtain the nonlinear equation

$$
T=V+T G^{(0)} T-T G^{(0)} V G^{(0)} T,
$$

which is then subtracted from the sum of Eqs. (2.23) and (2.26),

$$
T=V+V G^{(0)} T+T G^{(0)} V-T G^{(0)} T+T G^{(0)} V G^{(0)} T .
$$

The first variation of the left-hand side produced by small changes in $V$ on the right side is given by [56]

$$
\delta T=\left(V-T+T G^{(0)} V\right) G^{(0)} \delta T+\delta T G^{(0)}\left(V-T+V G^{(0)} T\right)=0,
$$

by virtue of Eqs. (2.23) and (2.26). Thus, if we start with a guess of the transition operator (e.g., the transition operator from a previous iteration of the DBIT algorithm) and insert it to the RHS of Eq. (2.36), the calculated $T$ will be a considerable improvement [56].

The above variational approach can in principle be used to estimate the overall transition operator for the whole model. Alternatively, the variational approach can be used to estimate the individual t-matrices of the different domains involved in the domain decomposition method described in the previous section. In any case, the main idea of the variational approach is to allow for iterative estimates of the transition operator that are not based on a perturbation expansion and, therefore, not based on any weak scattering assumptions [56]. 


\section{The nonlinear inverse scattering problem}

\subsection{The distorted Born iterative T-matrix inversion method}

The nonlinear inverse scattering problem in anisotropic elastic media is to estimate the stiffness tensor and mass density fields from measurements of the displacement-strain state vector $|\psi\rangle$ defined in Eq. (2.17) performed at the receiver surface. The nonlinear inverse scattering problem can be reduced to a sequence of linear inverse problems by using the distorted Born iterative T-matrix inversion (DBIT) method that can be described as follows: First, we invert the variation in the projection of the displacement-strain state vector onto the receiver surface $\langle r \mid \psi\rangle-\left\langle r \mid \psi^{(i)}\right\rangle$ for the variation in the scattering potential operator $\left(V^{(i+1)}-V^{(i)}\right)$ using a distorted Born approximation $[5,14,55,56,65]$ :

$$
\langle r \mid \psi\rangle-\left\langle r \mid \psi^{(i)}\right\rangle=\left\langle r\left|G^{(i)}\left(V^{(i+1)}-V^{(i)}\right)\right| \psi^{(i)}\right\rangle, \quad i=1,2, \cdots .
$$

The above equation represents the change in the data vector due to a small change in the model between two successive iterations. Here $G^{(i)}$ and $\left|\psi^{(i)}\right\rangle$ are the Green's operator and the wavefield corresponding to the scattering potential operator $V^{(i)}$ from the $i$ th iteration, which is given by Eq. (2.18) and assumed known; $V^{(i+1)}$ is the updated scattering potential matrix at iteration $i+1$. Then we update the displacement-strain state vector $|\psi\rangle$ within the model and the Green's function operator G using Eqs. (2.20) and (2.28)

$$
\begin{aligned}
& \left|\psi^{(i+1)}\right\rangle=\left|\psi^{(0)}\right\rangle+G^{(0)} T^{(i+1)}\left|\psi^{(0)}\right\rangle, \\
& G^{(i+1)}=G^{(0)}+G^{(0)} T^{(i+1)} G^{(0)},
\end{aligned}
$$

where

$$
T^{(i+1)}=\mathcal{T}\left(V^{(i+1)}\right)
$$

is the corresponding T-matrix. In Eq. (3.4), the $\mathcal{T}$-symbol denotes either the exact solution (2.25), the more variational estimate (2.36) and/or the domain decomposition method discussed in Subsection 2.3. Although the updated reference media in the above algorithm may be anisotropic, the sequence of estimated scattering potentials $V^{(i)}$ is defined relative to a fixed homogeneous isotropic elastic reference medium; which simplifies the parameterization of seismic anisotropy.

The distorted Born iterative T-matrix inversion (DBIT) method described above is fully consistent with the Gauss-Newton method [5], which is known to converge quadratically, provided that the initial model is sufficiently close to the true (unknown) model. However, our implementation of the Gauss-Newton method based on an integral equation description may be more attractive for certain applications than a conventional implementation based on finite differences and the adjoint state method. This is because our approach gives the Fréchet derivative operator directly in terms of the modified Green's function and strain field that can be updated after each iteration in an efficient manner based on domain decomposition and the variational methods discussed below. 


\subsection{Fréchet derivatives}

In accordance with common practice, we now assume that only the particle displacement vector data are available, so that the data residual equation (3.1) reduces to

$$
\langle r \mid u\rangle-\left\langle r \mid u^{(i)}\right\rangle=\omega^{2}\left\langle r\left|g^{(i)}\left(\Delta \rho^{(i+1)}-\Delta \rho^{(i)}\right)\right| u^{(i)}\right\rangle+\left\langle r\left|M^{(i)}\left(\Delta C^{(i+1)}-\Delta C^{(i)}\right)\right| \epsilon^{(i)}\right\rangle,
$$

where we have used Eqs. (2.17)-(2.18). In the real-space coordinate representation [5, 64], Eq. (3.5) becomes

$$
\begin{aligned}
u_{k}(\mathbf{r})-u_{k}^{(i)}(\mathbf{r})= & \omega^{2} \int d \mathbf{x} g_{k l}^{(i)}(\mathbf{r}, \mathbf{x})\left[\Delta \rho^{(i+1)}(\mathbf{x})-\Delta \rho^{(i)}(\mathbf{x})\right] u_{l}^{(i)}(\mathbf{x}) \\
& +\int d \mathbf{x} M_{k l m}^{(i)}(\mathbf{r}, \mathbf{x})\left[\Delta C_{l m n o}^{(i+1)}(\mathbf{x})-\Delta C_{l m n o}^{(i)}(\mathbf{x})\right] \epsilon_{n o}^{(i)}(\mathbf{x}),
\end{aligned}
$$

or

$$
\begin{aligned}
u_{k}(\mathbf{r})-u_{k}^{(i)}(\mathbf{r})= & \omega^{2} \int d \mathbf{x} g_{k l}^{(i)}(\mathbf{r}, \mathbf{x})\left[\Delta \rho^{(i+1)}(\mathbf{x})-\Delta \rho^{(i)}(\mathbf{x})\right] u_{l}^{(i)}(\mathbf{x}) \\
& +\int d \mathbf{x} M_{k L}^{(i)}(\mathbf{r}, \mathbf{x})\left[\Delta C_{L M}^{(i+1)}(\mathbf{x})-\Delta C_{L M}^{(i)}(\mathbf{x})\right] \epsilon_{M}^{(i)}(\mathbf{x})
\end{aligned}
$$

in the abbreviated subscript notation of Auld [66], where repeated upper case indices run from 1 to 6 . Here, we have also used Einstein's summation convention and the definition of stiffness and mass density perturbations given in Eqs. (2.4)-(2.5). Eq. (3.7) can be expressed more compactly as

$$
\begin{aligned}
& \delta u_{k}^{(i)}(\mathbf{r})=\omega^{2} \int d \mathbf{x} g_{k l}^{(i)}(\mathbf{r}, \mathbf{x}) \delta \rho^{(i+1)}(\mathbf{x}) u_{l}^{(i)}(\mathbf{x})+\int d \mathbf{x} M_{k L}^{(i)}(\mathbf{r}, \mathbf{x}) \delta C_{L M}^{(i+1)}(\mathbf{x}) \epsilon_{M}^{(i)}(\mathbf{x}), \\
& \delta u_{k}^{(i)}(\mathbf{r}) \equiv u_{k}(\mathbf{r})-u_{k}^{(i)}(\mathbf{r}) \\
& \delta \rho^{(i+1)}(\mathbf{x}) \equiv \Delta \rho^{(i+1)}(\mathbf{x})-\Delta \rho^{(i)}(\mathbf{x}) \\
& \delta C_{L M}^{(i+1)}(\mathbf{x}) \equiv \Delta C_{L M}^{(i+1)}(\mathbf{x})-\Delta C_{L M}^{(i)}(\mathbf{x})
\end{aligned}
$$

where $\delta u_{k}^{(i)}(\mathbf{r})$ is the data residual wavefield at the $i$ th iteration and $\delta C_{L M}^{(i+1)}(\mathbf{x})\left(\delta \rho^{(i+1)}(\mathbf{x})\right)$ is the variation in the stiffness elements (mass density) between two successive iterations.

The next step is to decompose the mass density and stiffness model perturbations as

$$
\Delta \rho(\mathbf{x})=\rho^{(0)}(\mathbf{x}) m^{(0)}(\mathbf{x}), \quad \Delta C_{L M}(\mathbf{x})=\sum_{p=1}^{21} B_{L M}^{(p)}(\mathbf{x}) m^{(p)}(\mathbf{x}),
$$

where $m^{(0)}(\mathbf{x})$ is a perturbation of the mass density normalized to the density of the reference medium, $m^{(p)}(\mathbf{x})$ represents the perturbation of one of the 21 elastic parameters and $B_{L M}^{(p)}(\mathbf{x})$ represents the tensor field structure related to the model (elastic) parameter $m^{(p)}$. The B-matrices in Eq. (3.12) associated with different elastic parameters depend on 
the parameterization of the model. In this study, we parameterize the model by using perturbations in the elastic stiffness elements that are normalized to the corresponding properties in an isotropic reference medium, or some other stiffness parameters. If $C_{K L}^{(0)}$ is non-zero, we use the following expression for the normalized stiffness perturbations

$$
m^{(p)}=\left(C_{K L}-C_{K L}^{(0)}\right) / C_{K L}^{(0)}, \quad K, L \rightarrow p=1, \cdots, 21,
$$

and a similar expression for the normalized mass density perturbations. By using model parameter perturbations that are normalized in this way, we ensure that the different (non-zero) model parameter perturbations are of approximately similar size, which is an advantage for multi-parameter FWI.

It follows from Eqs. (3.8)-(3.12) that

$$
\delta u_{k}^{(i)}(\mathbf{r})=\int d \mathbf{x} F_{k}^{(i, 0)}(\mathbf{r}, \mathbf{x}) \delta m^{(i+1, p)}(\mathbf{x})+\sum_{p=1}^{21} \int d \mathbf{x} F_{k}^{(i, p)}(\mathbf{r}, \mathbf{x}) \delta m^{(i+1, p)}(\mathbf{x}),
$$

where the full set of scalar and vectorial Fréchet derivatives after the $i$ th iteration is given by

$$
F_{k}^{(i, 0)}=\omega^{2} g_{k l}^{(i)}(\mathbf{r}, \mathbf{x}) \rho^{(0)} u_{l}^{(i)}(\mathbf{x}), \quad F_{k}^{(i, p)}(\mathbf{r}, \mathbf{x})=M_{k L}^{(i)}(\mathbf{r}, \mathbf{x}) B_{L M}^{(p)}(\mathbf{x}) \epsilon_{N}^{(i)}(\mathbf{x}), \quad p=1, \cdots, 21 .
$$

Note that $m^{(i, p)}$ represents the inverted perturbation in the $p$ th model parameter relative to a static reference medium, whereas $\delta m^{(i, p)}$ is the variation of this quantity between two successive iterations.

After a discretization of the seismic model involving $N_{r}$ receivers and $N$ grid blocks, Eq. (3.14) can be expressed as

$$
\delta \mathbf{u}^{(i)}=\sum_{p=0}^{21} \mathbf{F}^{(i, p)} \delta \mathbf{m}^{(i+1, p)},
$$

where $\operatorname{dim}\left(\delta \mathbf{u}^{(i)}\right)=3 N_{r}, \operatorname{dim}\left(\mathbf{F}^{(i, p)}\right)=3 N_{r} \times N$ and $\operatorname{dim}\left(\delta \mathbf{m}^{(i+1, p)}\right)=N \times N$.

Finally, we rewrite Eq. (3.16) in standard matrix form suitable for the application of linear inversion theory [67] at each iteration of the nonlinear DBIT algorithm as

$$
\delta \mathbf{u}^{(i)}=\mathbf{F}^{(i)} \delta \mathbf{m}^{(i+1)},
$$

where

$$
\mathbf{F}^{(i)}=\left(\mathbf{F}^{(i, 0)}, \mathbf{F}^{(i, 1)}, \cdots, \mathbf{F}^{(i, 21)}\right)
$$

and

$$
\delta \mathbf{m}^{(i)}=\left(\delta \mathbf{m}^{(i, 0)}, \delta \mathbf{m}^{(i, 1)}, \cdots, \delta \mathbf{m}^{(i, 21)}\right)^{T},
$$

are block-matrices of Fréchet-derivatives and model parameter perturbations, respectively.

The above form (3.17) of the data perturbation equation is convenient, since it allows us to use standard theory for solving linear inverse problems at each iteration of the distorted Born iterative method for nonlinear inversion. 


\subsection{Regularized least-squares solution for each linearized inversion step}

In order to estimate the model parameter perturbation vector $\delta m^{(i+1)}$ given an observation of the data residual vector $\delta u^{(i)}$ after the $i$ th iteration, we minimize the following objective function $[5,14]$

$$
O\left(\delta \mathbf{m}^{(i+1)}\right)=\left\|\delta \mathbf{u}_{o b s}^{(i)}-\mathbf{F}^{(i)} \delta \mathbf{m}^{(i+1)}\right\|^{2}+\left(\lambda^{(i)}\right)^{2}\left\|\delta \mathbf{m}^{(i+1)}\right\|^{2},
$$

where $\lambda^{(i)}$ is a regularization parameter depending on the iteration number, discussed at the end of this section. By minimizing the above objective function, we arrive at the following iterative algorithm for updating the model parameter vector $[4,5]$ :

$$
\mathbf{m}^{(i+1)}=\mathbf{m}^{(i)}+\left[\mathbf{H}^{(i)}+\left(\lambda^{(i)}\right)^{2} \mathbf{I}_{22 N}\right]^{-1} \mathbf{G}^{(i)},
$$

where $\mathbf{I}_{22 N}$ is the $22 N \times 22 N$ unit matrix,

$$
\mathbf{G}^{(i)}=\Re\left[\left(\mathbf{F}^{(i)}\right)^{\dagger} \delta \mathbf{u}^{(i)}\right]
$$

is the gradient vector (in the direction of steepest descent), and

$$
\mathbf{H}^{(i)}=\left(\mathbf{F}^{(i)}\right)^{\dagger} \mathbf{F}^{(i)}
$$

is the corresponding approximate Hessian matrix; which is very important for multiparameter FWI [39]. In Eqs. (3.22)-(3.23), the † symbol denotes the Hermitian conjugate. The selection of the optimal regularization parameter for nonlinear inverse scattering is challenging [5, 67-70]. Here we adjust the regularization parameter automatically after each iteration using a variant of the Levenberg-Marquard scheme for self-adaptive selection of the regularization parameter detailed by $[67,69]$. In this scheme, we start with an initial value of the regularization parameter derived by comparing deterministic and Bayesian inversion approaches [69]

$$
\lambda^{(0)}=\sqrt{\operatorname{mean}\left[\operatorname{diag}\left(\mathbf{H}^{(i)}\right)\right]} .
$$

The value of the regularization parameter at the $i$ th iteration is given by

$$
\lambda^{(i)}=a \lambda^{(i-1)},
$$

where $a$ is a constant that is smaller or larger than unity if the change in the relative residual data error $\epsilon_{d}$ between two successive iterations is negative or positive, respectively. The relative residual data error $\epsilon_{d}^{(i)}$ is defined by [5]

$$
\epsilon_{d}^{(i)}=\frac{\left\|\delta \mathbf{u}^{(i)}-\delta \mathbf{u}^{o b s}\right\|}{\left\|\delta \mathbf{u}^{o b s}\right\|} .
$$

According to Morozov's discrepancy principle $[14,68]$, the iterative nonlinear inversion procedure should preferably stop when $\epsilon_{d}$ approaches the noise level $\eta$. A pseudo code for implementation of the DBIT inversion algorithm in conjunction with the scheme for adaptive regularization parameter adjustment associated with Eqs. (3.21)-(3.26) and Morozov' discrepancy principle $[14,68]$ is given in Appendix C. 


\section{Numerical examples}

The DBIT algorithm for anisotropic elastic media developed in this study can in principle be used to invert for the mass density and elastic stiffness fields of arbitrary heterogeneous anisotropic media. However, we shall in the numerical experiments restrict ourselves to heterogeneous transversely isotropic (VTI) media with vertical symmetry axes with known mass density fields. As discussed by $[27,66]$, a VTI medium is generally characterized by the five independent Voigt stiffness parameters $C_{11}, C_{33}, C_{55}, C_{66}$, $C_{13}$. Since we have five independent elastic parameters to invert for at every point in space, the inverse scattering problem in VTI media is still very challenging, both due to the large memory requirements and CPU times required and due to the problem with cross-talk in the multi-parameter FWI $[33,37,38,40,42,44]$.

Although we shall use a normalized version of the Voigt stiffness parameterization (Eq. (3.13)), it is important to realize that the generalized DBIT algorithm we have developed can in principle be used with any parameterization of the seismic anisotropy, as long as one knows the corresponding B-matrices. The novelty of the present work is mainly related to our treatment of the nonlinear effects of multiple scattering (in the forward problem); which are taken into account by updating the (modified) Green's functions and wavefield within the model after each iteration by using the transition operator formalism (see Eqs. (3.2)-(3.4)).

\subsection{The three-layered VTI model}

We first apply the anisotropic elastic DBIT algorithm to the three-layered VTI model shown in Fig. 1, where a strongly anisotropic VTI medium is sandwiched between two weakly anisotropic VTI media. The values we have used for the different Voigt parameters are given in Table 3 in the paper of Jakobsen and Johansen [47], suggesting we are here using realistic values for the Voigt stiffness parameters based on ultrasonic laboratory measurements of shales samples from a borehole in the Brage field, North Sea. In our model parameterization based on Eq. (3.13), we employ a homogeneous isotropic reference medium with $\alpha=4270 \mathrm{~m} / \mathrm{s}, \beta=2735 \mathrm{~m} / \mathrm{s}$ and $\rho=2300 \mathrm{~kg} / \mathrm{m}^{3}$. Thus, the contrast between the initial model and the true (unknown) model is relatively large (See Fig. 1).

The three-layered test model, which is $2000 \mathrm{~m}$ wide and $500 \mathrm{~m}$ deep, was discretized into $80 \times 20$ grid blocks that are $25 \mathrm{~m}$ in each direction. The grid blocks take up the entire model in Fig. 1. Synthetic seismic waveform data in the frequency domain were generated for this model using a full integral equation solution in the frequency domain (Fig. 2). Frequency domain waveform data can be difficult to interpret, but they include diffractions and internal multiples in addition to travel time and amplitude data as well as turning rays. To avoid inverting unrealistically small data residuals, we added random Gaussian white noise to the computed waveform data. The signal-to-noise ratio was 60 $\mathrm{dB}$. We employed 80 sources and 80 receivers uniformly distributed along a single line at the top of the model. The sources were explosive and we used all three components of 

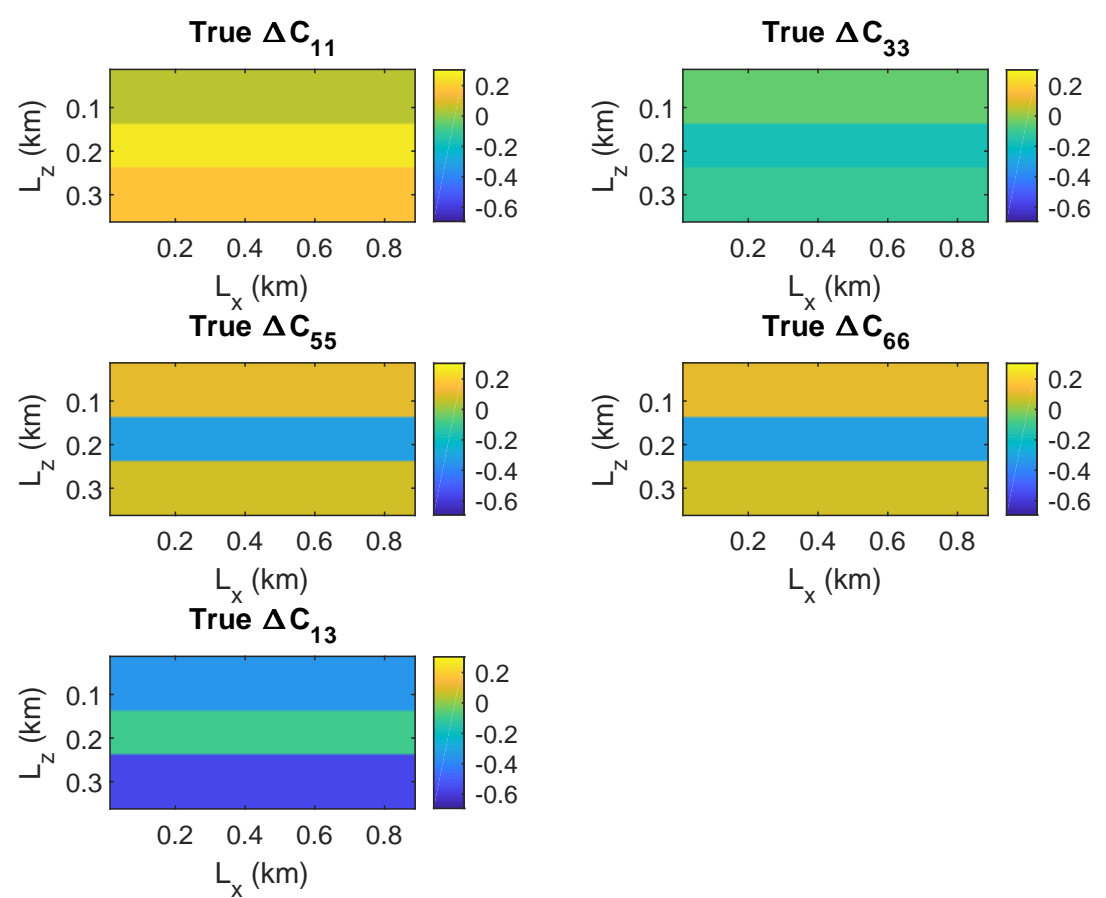

Figure 1: The true three-layered VTI model. Displayed are images of the perturbations in the different Voigt parameters normalized to the corresponding property in the homogeneous isotropic reference medium.
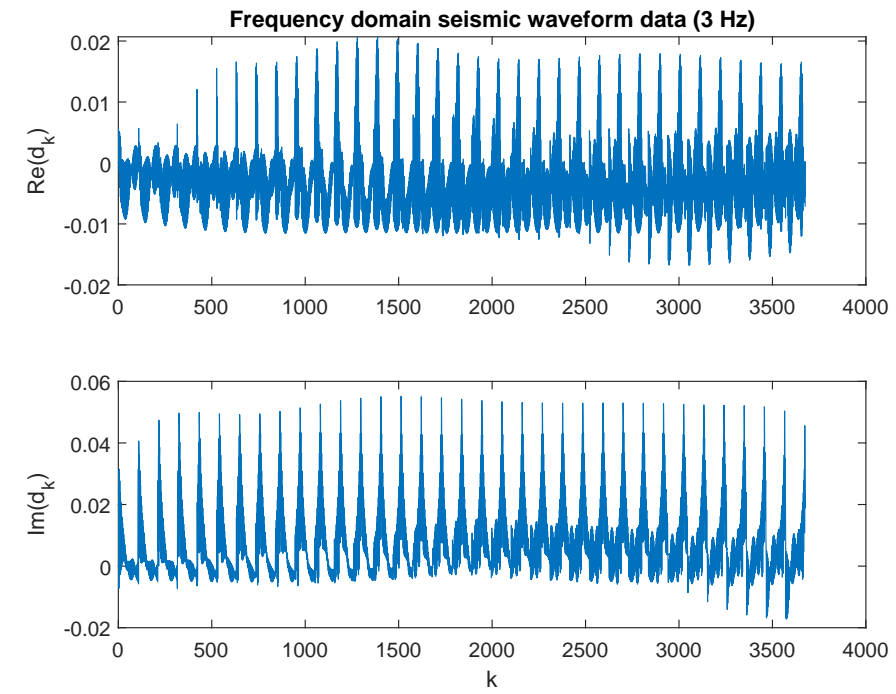

Figure 2: Synthetic waveform data corresponding to the true three-layered VTI model in Fig. 1 with the signalto-noise ratio $60 \mathrm{~dB}$. Displayed are the different components of the data vector $d_{k}$, which contain the three different components of particle displacement at all receiver positions for all source positions, organized into a single vector with components $k=1, \cdots, 3 N_{S} N_{r}$, where $N_{S}$ and $N_{r}$ are the numbers of sources and receivers, respectively. 

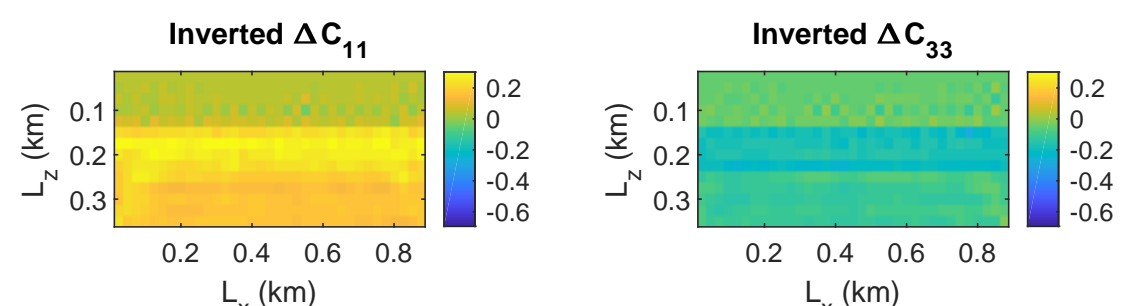

Inverted $\Delta \mathrm{C}_{55}$

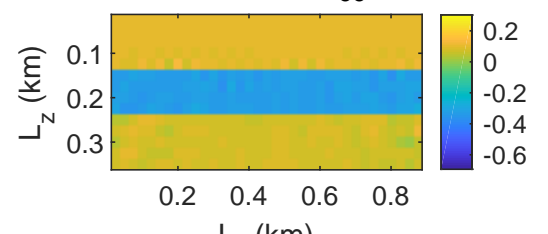

verted $\Delta \mathrm{C}_{66}$

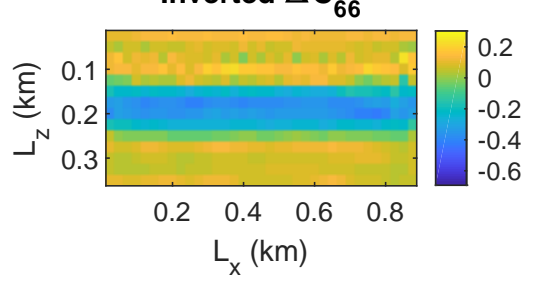

Inverted $\Delta \mathrm{C}_{13}$

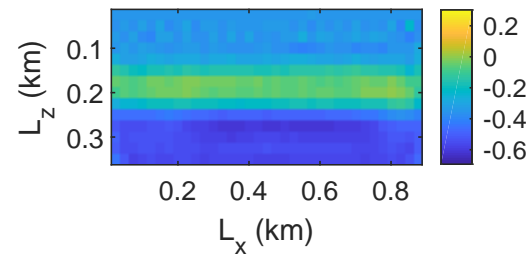

Figure 3: Results of anisotropic elastic FWI based on the DBIT algorithm for the synthetic waveform data shown in Fig. 2.

the particle displacement vector at the surface as data.

We performed a sequential inversion of data corresponding to $3 \mathrm{~Hz}, 5 \mathrm{~Hz}, 7.5 \mathrm{~Hz}, 10$ $\mathrm{Hz}$ and $15 \mathrm{~Hz}$ using the normalized version of the Voigt stiffnesses parameterization defined in Eq. (3.13). We carried out DBIT inversion using the workflow with self-adaptive selection of the regularization parameter described in Appendix C. The initial model was a homogeneous isotropic elastic medium identical to the isotropic elastic medium used in the parameterization of the model. In accordance with Morozov's discrepancy principle, we exited the iteration loop when the normalized data error became similar to the noise level (0.001). The inverted model is shown in the form of images and model vectors in Figs. 3 and 4, respectively. Clearly, one can see from Figs. 1, 3 and 4 that the inverted three-layer VTI model is very similar to the true three-layer VTI model. Fig. 5 illustrates the performance of the DBIT algorithm. Here one can see that both the overall model errors decreased monotonically down to their minimum values, leading to very encouraging initial inversion results for this simple test model.

\subsection{A resampled VTI Hess model}

It is necessary to investigate the performance of the anisotropic elastic DBIT algorithm for a more complicated and realistic model of the underground. We choose the resampled 


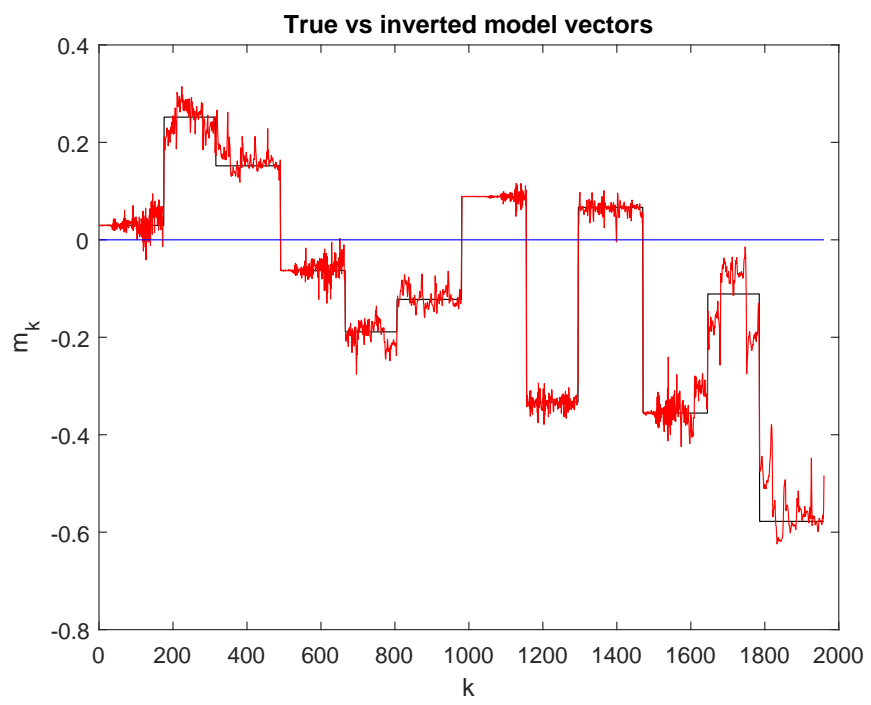

Figure 4: Results of anisotropic elastic FWI based on the DBIT algorithm for the synthetic waveform data shown in Fig. 2. Displayed is a comparison of the true model vector $m_{k}$ (straight lines) with the inverted model vector $m_{k}$ (oscillatory curve) obtained using the DBIT algorithm on the synthetic waveform data in Fig. 1. Also shown is the model parameter vector corresponding to the homogeneous isotropic initial model. The blue horizontal line represents the starting model.
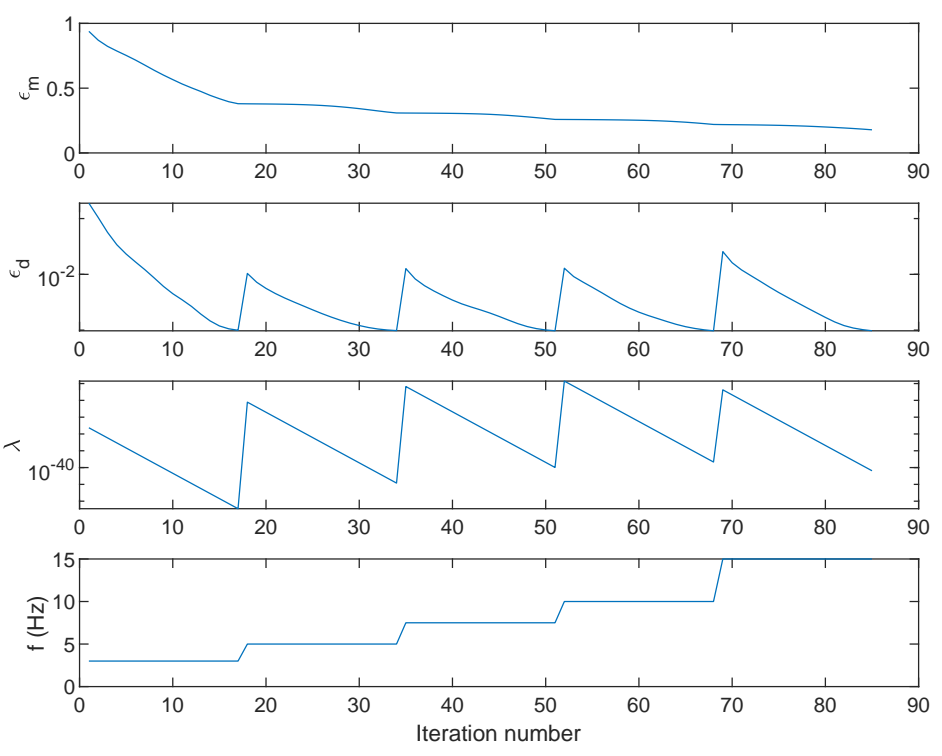

Figure 5: Performance of the DBIT algorithm for the layered VTI model shown in Fig. 1. Plots number 1 and 2 from the top show how the relative residual data $\epsilon_{d}$ and model errors $\epsilon_{m}$ vary with the number of iterations. Plots 3 and 4 from the top show the value of the regularization parameter $\lambda$ and the frequency $f$ used at the different iterations. The relative residual data error $\epsilon_{d}$ is defined in Eq. (3.26). The overall model inversion error $\epsilon_{m}$ at the $i$ th iteration is defined by $\left\|\mathbf{m}_{\text {true }}-\mathbf{m}_{i}\right\| /\left\|\mathbf{m}_{\text {true }}\right\|$. 

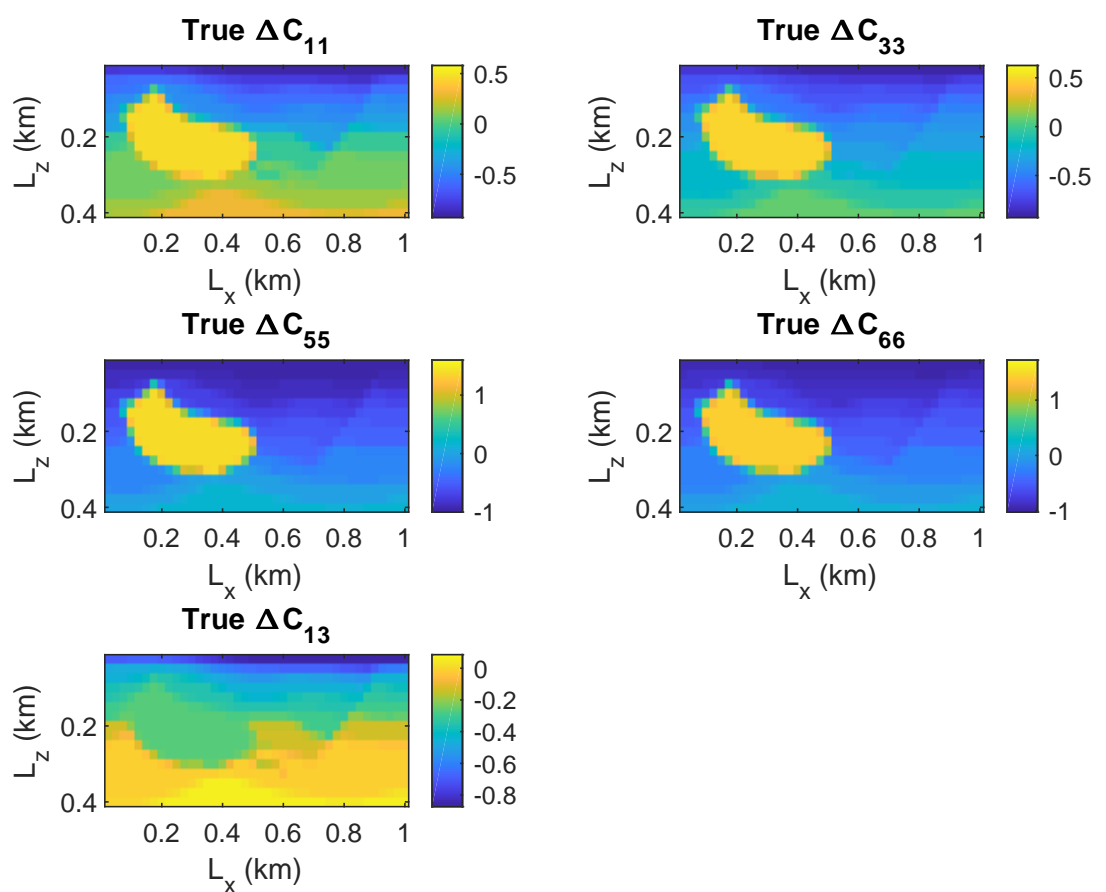

Figure 6: The true VTI Hess model. Displayed are images of the non-dimensional perturbation in the different Voigt parameters normalized to the corresponding property in the homogeneous isotropic reference medium.

version of the VTI Hess model shown in Fig. 6; which is laterally inhomogeneous and includes a high-velocity salt body as well as several faults and layers with different VTI elastic properties. We employ an arbitrary homogeneous isotropic reference medium with arbitrary but typical velocities $\alpha=3652 \mathrm{~m} / \mathrm{s}$ and $\beta=1778 \mathrm{~m} / \mathrm{s}$ and mass density $\rho=2300 \mathrm{~kg} / \mathrm{m}^{3}$. This implies that the difference between the initial model and the true (unknown) model is relatively large (Fig. 6). The resampled Hess VTI model, which is $1000 \mathrm{~m}$ wide and 400 deep, was discretized into $40 \times 16$ grid blocks that are $25 \mathrm{~m}$ in each direction. The grid blocks take up the entire model in Fig. 6.

Synthetic seismic waveform data in the frequency domain were generated for this model using a full integral equation solution in the frequency domain (not shown). We first added random Gaussian white noise to the computed waveform data, so that the signal-to-noise ratio was $80 \mathrm{~dB}$ and $40 \mathrm{~dB}$ in two different numerical experiments. We employed 40 sources and 40 receivers uniformly distributed along a single line at the top of the model. The sources were explosive and we used all three components of the particle displacement vector at the surface as data.

Again, we performed a sequential inversion of data corresponding to $3 \mathrm{~Hz}, 5 \mathrm{~Hz}, 7.5$ $\mathrm{Hz}, 10 \mathrm{~Hz}$ and $15 \mathrm{~Hz}$ using the normalized version of the Voigt stiffnesses parameterization defined in Eqs. (3.13). We employed the anisotropic elastic DBIT algorithm with self-adaptive selection of the regularization parameter described in Appendix C. The ini- 

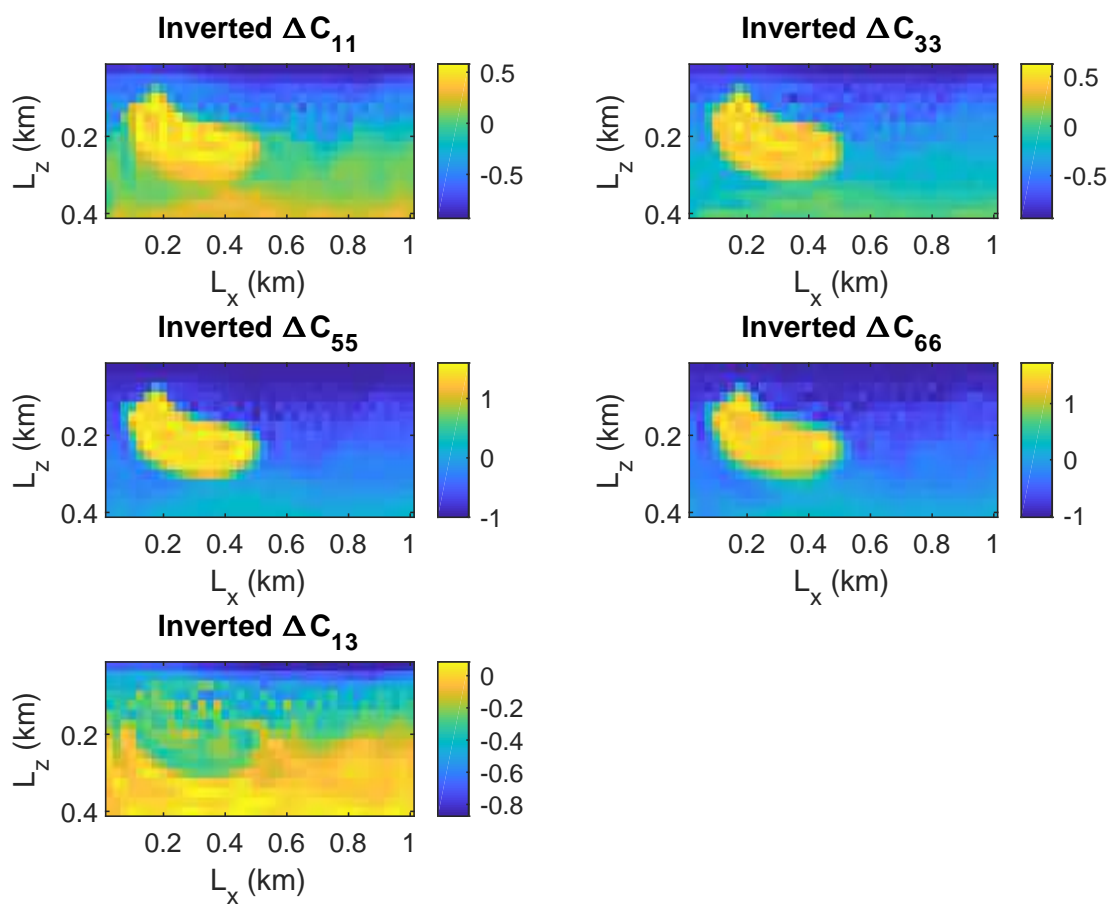

Figure 7: Results of anisotropic elastic FWI based on the DBIT algorithm for the VTI Hess model in Fig. 6 when the signal-to-noise ratio is $80 \mathrm{~dB}$.

tial model was a homogeneous isotropic elastic medium identical to the isotropic elastic medium used in the parameterization of the model. In accordance with Morozov's discrepancy principle, we exited the iteration loop when the normalized data error became similar to the noise level. The inverted models for signal-to-noise ratio equal to $80 \mathrm{~dB}$ or $40 \mathrm{~dB}$ (noise levels equal to 1e-4 and 1e-3) are shown in the form of images in Figs. 7 and 8 , respectively. Clearly, one can see from Figs. 6, 7 and 8 that the inverted VTI Hess models at $80 \mathrm{~dB}$ and $40 \mathrm{~dB}$ signal-to-noise ratios are very similar to the true VTI Hess model, and that the results are stable with respect to increments in the noise. It is interesting to see that the parts of the VTI Hess model with large contrasts in the elastic parameters (e.g., the salt body) are better reconstructed than the interfaces with weaker contrasts in the anisotropic elastic properties. Figs. 9 and 10 illustrates the behaviour of the anisotropic elastic DBIT algorithm at $80 \mathrm{~dB}$ and $40 \mathrm{~dB}$ signal-to-noise ratio. Since the inversion is terminated when the data error becomes equal to the noise level, it should not come as a surprise that total computation time is larger at $80 \mathrm{~dB}$ than at $40 \mathrm{~dB}$ signal-to-noise ratio. In both Figs. 9 and 10, the relative residual data error is seen to decrease monotonically with increasing number of iteration, except from the last three iterations in the case of 80 $\mathrm{dB}$. The overall model error is seen to decrease monotonically with the iteration number and to remain stable when the data error starts to increase. 


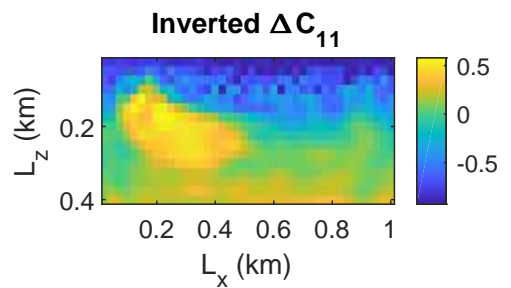

Inverted $\Delta \mathrm{C}_{55}$

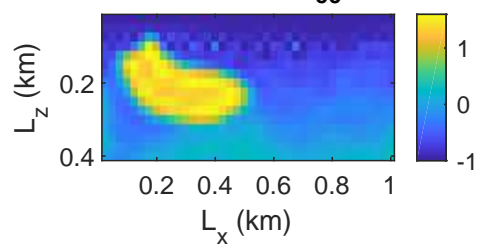

Inverted $\Delta \mathrm{C}_{13}$

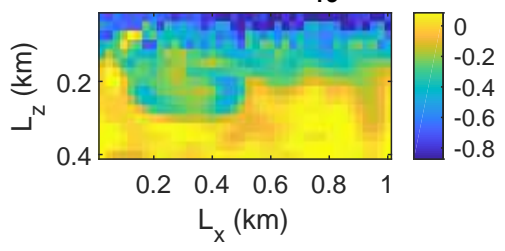

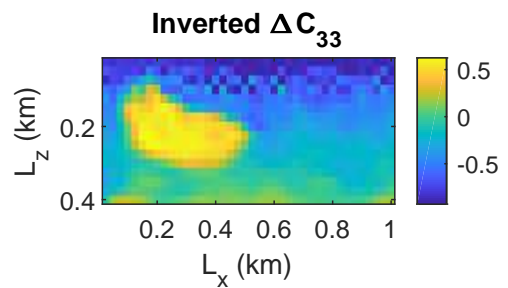

Inverted $\Delta \mathrm{C}_{66}$

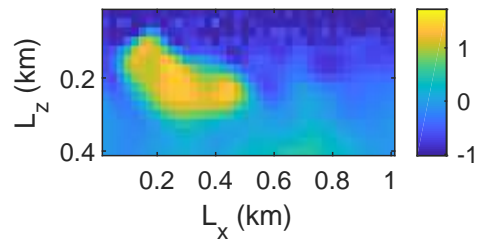

Figure 8: Results of anisotropic elastic FWI based on the DBIT algorithm for the VTI Hess model in Fig. 6 when the signal-to-noise ratio is $40 \mathrm{~dB}$.
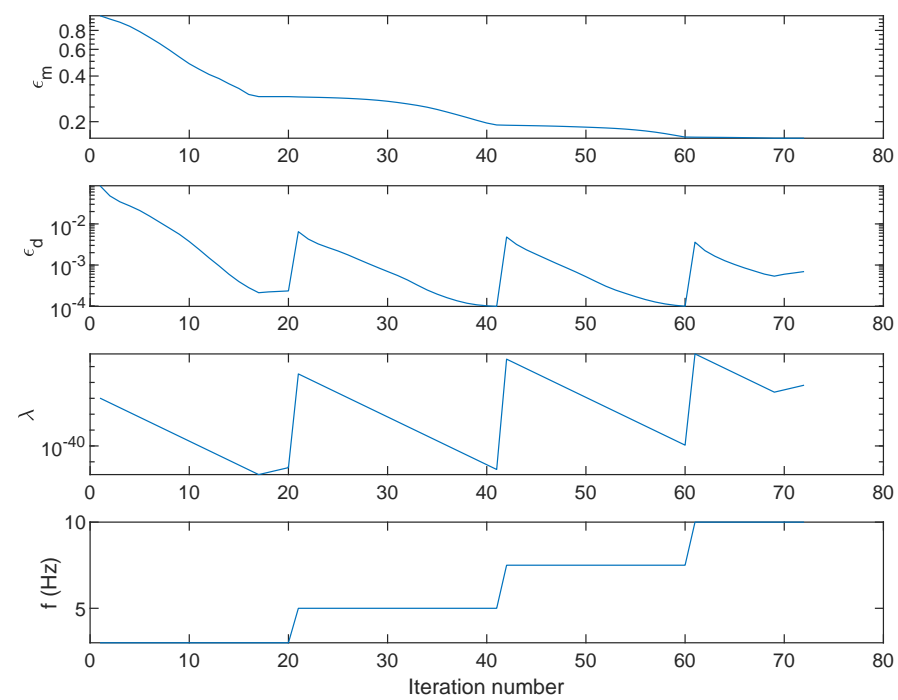

Figure 9: Performance of the DBIT algorithm for the true VTI Hess model shown in Fig. 6 when the signalto-noise ratio is $80 \mathrm{~dB}$. Plots number 1 and 2 from the top show how the relative residual data $\epsilon_{d}$ and model errors $\epsilon_{m}$ vary with the number of iterations. Plots 3 and 4 from the top show the value of the regularization parameter $\lambda$ and the frequency $f$ used at the different numbers of iterations. The total CPU-time including the generation of synthetic seismic waveform data for selected frequencies were $118.4 \mathrm{~min}$. 

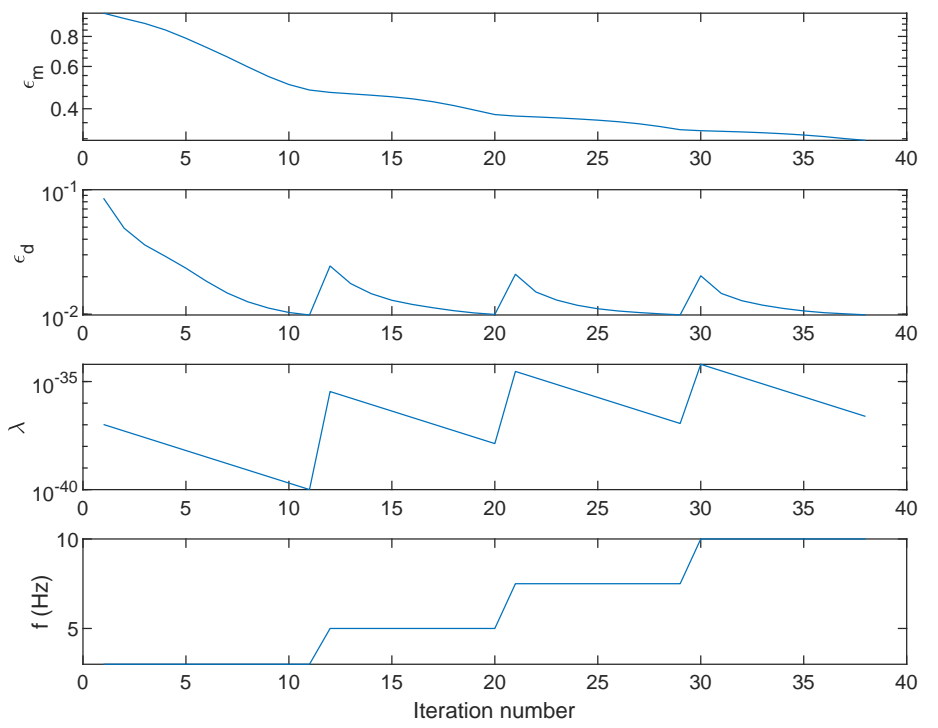

Figure 10: Performance of the DBIT algorithm for the true VTI Hess model shown in Fig. 6 when the signalto-noise ratio is $40 \mathrm{~dB}$. Plots number 1 and 2 from the top show how the relative residual data $\epsilon_{d}$ and model errors $\epsilon_{m}$ vary with the number of iterations. Plots 3 and 4 from the top show the value of the regularization parameter $\lambda$ and the frequency $f$ used at the different numbers of iterations. The total CPU-time including the generation of synthetic seismic waveform data for selected frequencies were $84.1 \mathrm{~min}$.

\section{Concluding remarks}

We have generalized the distorted Born iterative T-matrix (DBIT) method that Jakobsen and Ursin [5] developed on the basis of the scalar wave equation so that it can be used in conjunction with the elastodynamic equation for an arbitrary anisotropic elastic continuum with variable mass density as well as elastic stiffnesses. The theory is general, but the numerical results were performed for laterally homogeneous and inhomogeneous VTI media with known mass density. We found a good match between the true and inverted models using a normalized Voigt stiffness parameterization and we think that the use of approximate Hessian information was essential for the good performance of our Gauss-Newton consistent DBIT algorithm. We used the exact solution (2.25) to update the transition operator after each iteration, but we have found that the variational estimate (2.36) gives practically identical results. The memory requirements and computational cost scales like $N^{2}$ and $N^{3}$, respectively. Therefore, future research will focus on the acceleration of the generalized DBIT method presented here, for example, by involving the use of Fast Fourier transforms for the calculation of certain convolution integrals and the use of hierarchical matrices for the computation of the inverse Hessian for large models. The anisotropic elastic FWI method presented here may be particularly suitable for time-lapse studies, since the inversion can then be focused on a relatively small 
target-domain (a reservoir under production) under the assumption that the background model is known. The fact that the DBIT method involves an explicit expression for the sensitivity matrix in terms of modified Green's functions and strain fields will become an advantage when it comes to the estimation of uncertainties via Bayesian inversion methods. Our plans for future research include numerical experiments on lower anisotropy symmetry media and applications to seismic fracture characterization as well as timelapse studies.

\section{A Reference Green's function and it's spatial derivatives}

\section{A.1 Ray-theory (modified) Green's function}

Assuming that there are no caustics, ray-theory Green's function $\mathbf{G}$ at the receiver $R$ generated at the source $S$ in a vertically inhomogeneous isotropic medium, in which $c$ and $\rho$ are the space-dependent $\mathrm{P}$ - or S-wave velocities and density, reads:

$$
G_{i n}(R, S ; \omega)=\frac{A(R) g_{i}(R) g_{n}(S) \exp [\mathrm{i} \omega \tau(R, S)]}{4 \pi[\rho(S) \rho(R) c(S) c(R)]^{1 / 2}} .
$$

Here $\tau(R, S)$ is the traveltime from $S$ to $R, A(R)$ is the scalar ray amplitude at $R, \mathbf{g}(X)$ is a unit polarization vector at $X$ and $\omega$ is a circular frequency. To construct the derivative of the ray-theory Green's function, we need to know the slowness vectors $\mathbf{p}(S)$ and $\mathbf{p}(R)$. They are given by

$$
\mathbf{p}(S)=\mathbf{g}(S) / c(S), \quad \mathbf{p}(R)=\mathbf{g}(R) / c(R) .
$$

The first derivative $G_{i n, k}(R, S ; \omega)$ of the Green's function with respect to $x_{k}(S)$ then reads

$$
G_{i n, k}(R, S ; \omega)=\mathrm{i} \omega G_{i n}(R, S ; \omega) p_{k}(S) .
$$

Similarly, the derivative $G_{i n, l}(R, S ; \omega)$ of the Green's function with respect to $x_{l}(R)$ reads

$$
G_{i n, l}(R, S ; \omega)=\mathrm{i} \omega G_{i n}(R, S ; \omega) p_{l}(R) .
$$

As an example, here is the second derivative $G_{i n, k l}(R, S ; \omega)$ of the Green's function with respect to $x_{k}(S)$ and $x_{l}(S)$ :

$$
G_{i n, k l}(R, S ; \omega)=-\omega^{2} G_{i n}(R, S ; \omega) p_{k}(S) p_{l}(S) .
$$

The other second derivatives can be obtained in a similar way. For each wave, one needs to know the traveltime and the ray amplitude, $\tau$ and $A$, and two unit vectors, $\mathbf{t}(S)$ and $\mathbf{t}(R)$, tangent to the ray at $S$ and $R$, calculated by using a computer program like for example ANRAY [71]. In case of $\mathrm{P}$ wave, one can specify a polarization vector $\mathbf{g}$ as follows:

$$
\mathbf{g}^{(P)} \equiv\left(t_{1}, t_{2}, t_{3}\right)
$$


In case of $\mathbf{S}$ wave, one can specify polarization vectors $\mathbf{g}$ as follows:

$$
\begin{aligned}
& \mathbf{g}^{(S 1)} \equiv D^{-1}\left(t_{1} t_{3}, t_{2} t_{3},-D^{2}\right), \quad \mathbf{g}^{(S 2)} \equiv D^{-1}\left(-t_{2}, t_{1}, 0\right) \\
& D=\left(t_{1}^{2}+t_{2}^{2}\right)^{1 / 2}, \quad t_{1}^{2}+t_{2}^{2}+t_{3}^{2}=1
\end{aligned}
$$

Here $t_{i}$ are components of the unit vector $\mathbf{t}$. This way of the determination of polarization vector is possible in homogeneous and vertically inhomogeneous media. In generally heterogeneous media, it will be necessary to provide S-wave polarization vectors at $R$ using a computer program like for example ANRAY [71].

\section{A.2 Leading-term (modified) ray-theory Green's function}

The classic ray-theory formulas for the (modified) Green's function given above are relatively simple but correspond to the far-field approximation. Since near-field effects can obviously be important for multiple scattering within a seismic model, we derive here new results that are nearly as accurate as the exact formulas, but much simpler and more suitable for semi-analytical calculations.

In a homogeneous isotropic medium, the exact Green's function $\mathrm{G}$ can be expressed in the form of a three-term ray series. The ray series can be split into two parts, one corresponding to $\mathrm{P}$ waves, the other to $\mathrm{S}$ waves. It was shown that the dominant role in each of the series (for P and S waves) is played by two terms. The first term, the zeroorder term represents the well-known far-field approximation. The second term, in the ray theory called the additional term, represents a part of the first-order term of the ray series. It is the part, which contributes to the direction perpendicular to the polarization of the zero-order term. In the case of $\mathrm{P}$ waves, the additional term is perpendicular to the direction of the propagation of the $\mathrm{P}$ wave, in the case of $S$ waves, the additional term is parallel to the direction of the propagation. Together, the two above-described terms are called the leading term of the ray series. As [72] shows, the accuracy of the leading term does not differ much from the complete (exact) Green's function. Because of its importance, we concentrate on the leading term only, in the following.

\section{A.2.1 Leading-term Green's functions for P-and S-waves}

In the case of a homogeneous elastic isotropic medium, the leading term has the form [72]:

$$
G_{k l}(r, \omega)=G_{k l}^{P}(r, \omega)+G_{k l}^{S}(r, \omega),
$$

where

$$
G_{k l}^{P}(r, \omega) \sim \frac{A(r)}{r}\left(N_{k} N_{l}+\frac{\alpha}{\mathrm{i} \omega r} e_{J k} e_{j l}\right)
$$

and

$$
G_{k l}^{S}(r, \omega) \sim \frac{B(r)}{r}\left(e_{j k} e_{j l}+\frac{\beta}{i \omega r} N_{k} N_{l}\right)
$$


In Eqs. (A.10) and (A.11), $\alpha$ and $\beta$ are P- and S-wave velocities, $r$ is the source-receiver distance, vector $\mathbf{N}$ is identical with the vector $\mathbf{g}^{(P)}$ defined in Eq. (A.6) and vectors $\mathbf{e}_{1}$ and $\mathbf{e}_{2}$ are identical to vectors (A.7). Lower-case indices run from 1 to 3 , upper-case indices run from 1 to 2 . Einstein summation rule is applied. The terms $A(r)$ and $B(r)$ are defined as

$$
A(r)=\frac{\exp (\mathrm{i} \omega r / \alpha)}{4 \pi \rho \alpha^{2}}
$$

and

$$
B(r)=\frac{\exp (\mathrm{i} \omega r / \beta)}{4 \pi \rho \beta^{2}}
$$

\section{A.2.2 Modified Green's function for P-waves}

We concentrate first on the $G_{k l}^{P}$ part of the Green's function. Eq. (A.10) can be rewritten to the following form:

$$
G_{k l}^{P}(r, \omega) \sim \frac{A(r)}{r^{2}}\left[a \delta_{k l}+\frac{x_{k} x_{l}}{r}\left(1-\frac{a}{r}\right)\right]
$$

where

$$
a=\alpha / \mathrm{i} \omega .
$$

Let us introduce the following notation:

$$
X(r)=A(r), \quad Y_{k l}(r)=a \delta_{k l}+\frac{x_{k} x_{l}}{r}\left(1-\frac{a}{r}\right), \quad Z(r)=r^{2} .
$$

Eq. (A.14) can be then rewritten as

$$
G_{k l}^{P}(r, \omega)=\frac{X Y_{k l}}{Z}
$$

From Eq. (A.17), we obtain for the first and second spatial derivatives of $G^{P}$, the following expressions:

$$
G_{k l, i}^{P}=\frac{\left(X, i Y_{k l}+X Y_{k l, i}\right) Z-X Y_{k l} Z_{, i}}{Z^{2}}
$$

and

$$
\begin{aligned}
G_{k l, i j}^{P}= & \frac{X_{, i j} Y_{k l}+X Y_{k l, i j}+X_{, i} Y_{k l, j}+X_{, j} Y_{k l, i}}{Z} \\
& -\frac{Z_{, i j} X Y_{k l}+X_{, i} Z_{, j} Y_{k l}+Y_{k l, i} Z_{, j} X+X_{, j} Z_{, i} Y_{k l}+Y_{k l, j} Z_{, i} X}{Z^{2}}+2 \frac{Z_{, i} Z_{, j} X Y_{k l}}{Z^{3}} .
\end{aligned}
$$

The first and second spatial derivatives of $X, Y_{k l}$ and $Z$ read:

$$
X_{, i}=A_{, i}=A \frac{\mathrm{i} \omega x_{i}}{\alpha r}=\frac{A}{a} \frac{x_{i}}{r}
$$




$$
\begin{aligned}
X_{, i j}= & A_{, i j}=A\left[\frac{\mathrm{i} \omega}{\alpha r}\left(\delta_{i j}-\frac{x_{i} x_{j}}{r^{2}}\right)-\frac{\omega^{2} x_{i} x_{j}}{\alpha^{2} r^{2}}\right]=\frac{A}{a}\left[\frac{1}{r}\left(\delta_{i j}-\frac{x_{i} x_{j}}{r^{2}}\right)+\frac{x_{i} x_{j}}{a r^{2}}\right], \\
Y_{k l, i}= & \frac{\delta_{i k} x_{l}+\delta_{i l} x_{k}}{r}\left(1-\frac{a}{r}\right)-\frac{x_{k} x_{l} x_{i}}{r^{3}}\left(1-2 \frac{a}{r}\right), \\
Y_{k l, i j}= & \frac{\delta_{i k} \delta_{j l}+\delta_{i l} \delta_{j k}}{r}\left(1-\frac{a}{r}\right) \\
& -\frac{\delta_{i k} x_{l} x_{j}+\delta_{i l} x_{j} x_{k}+\delta_{j k} x_{l} x_{i}+\delta_{j l} x_{k} x_{i}+\delta_{i j} x_{k} x_{l}}{r^{3}}\left(1-2 \frac{a}{r}\right)+\frac{x_{k} x_{l} x_{i} x_{j}}{r^{5}}\left(3-8 \frac{a}{r}\right), \\
Z_{, i}= & 2 x_{i}, \\
Z_{, i j}= & 2 \delta_{i j} .
\end{aligned}
$$

\section{A.2.3 Modified Green's function for S-waves}

Let us now concentrate on $\mathrm{S}$ waves (in elastic, isotropic and homogeneous media). Eq. (A.11) for $G_{k l}^{S}$ part of the Green's function can be rewritten to the form:

$$
G_{k l}^{S}(r, \omega) \sim \frac{B(r)}{r}\left[\delta_{k l}+\frac{x_{k} x_{l}}{r^{2}}\left(\frac{2 b}{r}-1\right)\right]
$$

where

$$
b=\beta / \mathrm{i} \omega .
$$

Let us introduce the following notation:

$$
V(r)=B(r), \quad W_{k l}(r)=\delta_{k l}+\frac{x_{k} x_{l}}{r^{2}}\left(\frac{2 b}{r}-1\right), \quad U(r)=r .
$$

Eq. (A.26) can be then rewritten as

$$
G_{k l}^{S}(r, \omega)=\frac{V W_{k l}}{U}
$$

From Eq. (A.29), we obtain for the first and second spatial derivatives of $G^{S}$, the following expressions:

$$
G_{k l, i}^{S}=\frac{\left(V_{, i} W_{k l}+V W_{k l, i}\right) U-V W_{k l} U_{, i}}{U^{2}}
$$

and

$$
\begin{aligned}
G_{k l, i j}^{S}= & \frac{V_{, i j} W_{k l}+V W_{k l, i j}+V_{, i} W_{k l, j}+V_{, j} W_{k l, i}}{U} \\
& -\frac{U_{, i j} V W_{k l}+V_{, i} U_{, j} W_{k l}+U_{, j} W_{k l, i} V+V_{, j} U_{, i} W_{k l}+W_{k l, j} U_{, i} V}{U^{2}}+2 \frac{U_{, i} U_{, j} V W_{k l}}{U^{3}} .
\end{aligned}
$$


The first and second spatial derivatives of $V, W_{k l}$ and $U$ read:

$$
\begin{aligned}
V_{, i}= & B, i=B \frac{\mathrm{i} \omega x_{i}}{\beta r}=\frac{B}{b} \frac{x_{i}}{r} \\
V_{, i j}= & B, i j=B\left[\frac{\mathrm{i} \omega}{\beta r}\left(\delta_{i j}-\frac{x_{i} x_{j}}{r^{2}}\right)-\frac{\omega^{2} x_{i} x_{j}}{\beta^{2} r^{2}}\right]=\frac{B}{b}\left[\frac{1}{r}\left(\delta_{i j}-\frac{x_{i} x_{j}}{r^{2}}\right)+\frac{x_{i} x_{j}}{r^{2}}\right] \\
W_{k l, i}= & \frac{\delta_{i k} x_{l}+\delta_{i l} x_{k}}{r^{2}}\left(\frac{2 b}{r}-1\right)-2 \frac{x_{k} x_{l} x_{i}}{r^{4}}\left(\frac{3 b}{r}-1\right) \\
W_{k l, i j}= & \frac{\delta_{i k} \delta_{j l}+\delta_{i l} \delta_{j k}}{r^{2}}\left(\frac{2 b}{r}-1\right) \\
& -2 \frac{\delta_{i k} x_{l} x_{j}+\delta_{i l} x_{j} x_{k}+\delta_{j k} x_{l} x_{i}+\delta_{j l} x_{k} x_{i}+\delta_{i j} x_{k} x_{l}}{r^{4}}\left(\frac{3 b}{r}-1\right)+2 \frac{x_{k} x_{l} x_{i} x_{j}}{r^{6}}\left(\frac{15 b}{r}-4\right), \\
U_{, i}= & \frac{x_{i}}{r} \\
U_{, i j}= & \frac{1}{r}\left(\delta_{i j}-\frac{x_{i} x_{j}}{r^{2}}\right) .
\end{aligned}
$$

\section{B Derivation of Eq. (2.25)}

The transition operator $T$ is given by Eq. (2.24):

$$
T=\left(I-V G^{(0)}\right)^{-1} V \text {. }
$$

By inverting the above Eq. (B.1) we obtain

$$
T^{-1}=V^{-1}\left(I-V G^{(0)}\right),
$$

or

$$
T^{-1}=V^{-1}-G^{(0)} .
$$

By operating on Eq. (B.3) with $V$ from the right, we obtain

$$
T^{-1} V=I-G^{(0)} V .
$$

Inversion of the above Eq. (B.4) yields

$$
V^{-1} T=\left(I-G^{(0)} V\right)^{-1}
$$

Finally, we operate on Eq. (B.5) with $V$ from the right and obtain

$$
T=V\left(I-G^{(0)} V\right)^{-1},
$$

which is identical with Eq. (2.25). 


\section{DBIT algorithm for anisotropic elastic media}

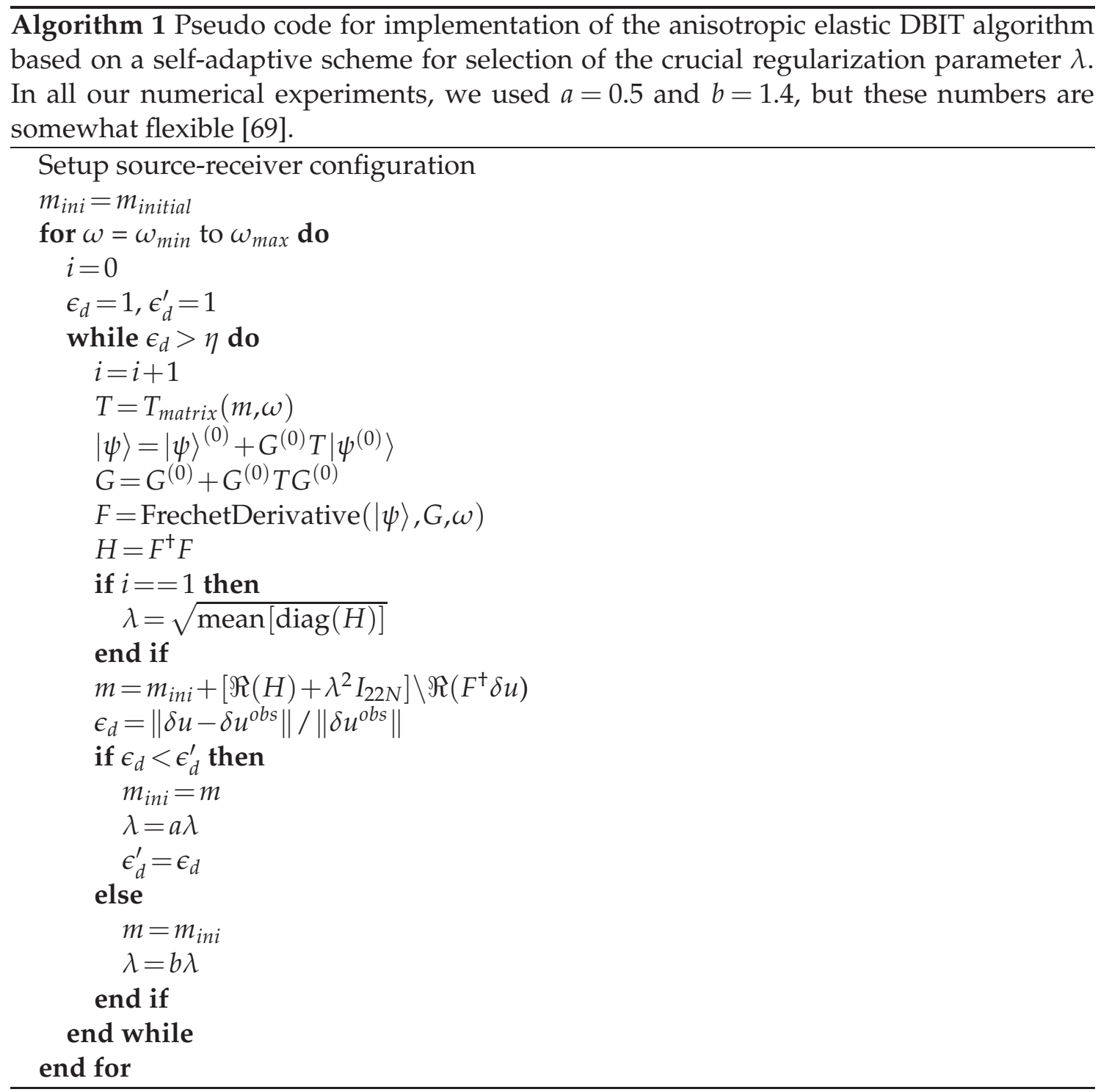

\section{References}

[1] P. Lailly, The Seismic Inverse Problem as a Sequence of Before Stack Migrations. In Proc. of the Conf. on Inverse Scattering: Theory and Applications (Philadelphia: SIAM), 1983.

[2] A. Tarantola, A. Inversion of seismic reflection data in the acoustic approximation, Geophysics, 49 (1984), 1259-1266. 
[3] R.G. Pratt, C. Shin, and G.J. Hicks, Gauss-Newton and full Newton methods in frequencyspace seismic waveform inversion, Geophysical Journal International, 133 (1998), 341-362.

[4] J. Virieux and S. Operto, An overview of full waveform inversion in exploration geophysics, Geophysics, 74 (2009), WCC127-WCC152.

[5] M. Jakobsen and B. Ursin, Full waveform inversion in the frequency domain using direct iterative T-matrix methods, Journal of Geophysics and Engineering, 12 (2015), 400-418.

[6] C. Bunks, F.M. Salech, S. Zaleski and G. Chavent, Multiscale seismic waveform inversion, Geophysics, 60 (1995), 1457-1473.

[7] R.G. Pratt, Seismic waveform inversion in the frequency domain, Part 1: theory and verification in a physical scale model, Geophysics, 64 (1999), 888-901.

[8] R.S. Wu and Y. Zheng, Nonlinear partial derivative and its De Wolf approximation for nonlinear seismic inversion, Geophysical Journal International, 196 (2014), 1827-1843.

[9] R.S. Wu, J. Luo and B. Wu, Seismic envelope inversion and modulation signal model, Geophysics, 79 (2014), WA13-WA24.

[10] D.J. Kouri and A. Vijay, Inverse scattering theory: renormalization of the LippmannSchwinger equation for acoustic scattering in one dimension, Physical Review Letters, 67 (2003), 046614,1-12.

[11] M. Jakobsen and R.S. Wu, Renormalized scattering series for frequency domain waveform modelling in strongly scattering media, Geophysical Journal International, 206 (2016), 880899.

[12] J.R. Krebs, J.E. Anderson, D. Hinkley, R. Neelamani, S. Lee, A. Baumsten and M. Lacasse, Fast full waveform seismic inversion using encoded sources, Geophysics, 74 (2009), WCC177-WCC188.

[13] A. Malcholm and B. Willemsen, Rapid 4D FWI using a local solver, The Leading Edge, 35 (2016), 1053-1059.

[14] M. Jakobsen and R.S. Wu, Accelerating the T-matrix approach to seismic full waveform inversion by domain decomposition, Geophysical Prospecting, 66 (2018), 1039-1059.

[15] V. Prieux, R. Brossier, S. Operto and J. Virieux, Multiparameter full waveform inversion of multicomponent ocean-bottom cable data from the Valhall field. Part 1: Imaging compressional wave speed, density and attenuation, Geophysical Journal International, 194 (2013), 1640-1664.

[16] L. Métivier, R. Brossier, S. Operto and J. Virieux, Acoustic multi-parameter FWI for the reconstruction of P-wave velocity, density and attenuation: Preconditioned truncated Newton approach, 85th Annual International Meeting, SEG, Expanded Abstracts, (2015), 1198-1203.

[17] T. Alkhalifah, Research note: insights into the data dependency on anisotropy: an inversion prospective, Geophysical Prospecting, 64 (2016), 505-513.

[18] O.C. Agudo, N.V. Silva, M. Warner and M. Morgan, Acoustic full-waveform inversion in an elastic world, Geophysics, 83 (2018), R257-R271.

[19] V. Prieux, R. Brossier, Y. Gholami, S. Operto, J. Virieux, O.I. Barkved and J.H. Kommedal, On the footprint of anisotropy on isotropic full waveform inversion: the Valhall case study, Geophysical Journal International, 187 (2011), 1495-1515.

[20] A. Tarantola, A strategy for nonlinear elastic inversion of seismic reflection data, Geophysics, 51 (1986), 1893-1903.

[21] P. Mora, Nonlinear two-dimensional elastic inversion of multioffset seismic data, Geophysics, 52 (1987) 1211-1228.

[22] R. Brossier, S. Operto and J. Virieux, Seismic imaging of complex onshore structures by 2D elastic frequency-domain full-waveform inversion, Geophysics, 74 (2009), WCC105- 
WCC118.

[23] D. Vigh, K. Jiao, D. Watts and D. Sun, Elastic full-waveform inversion application using multicomponent measurements of seismic data collection, Geophysics, 79 (2014), R63-R77.

[24] E.B. Raknes, B. Arntzen and W. Weibull, Three-dimensional elastic full waveform inversion using seismic data from the Sleipner area, Geophysical Journal International, 202 (2015), 1877-1894.

[25] E.B. Raknes and W. Weibull, Efficient 3D elastic full-waveform inversion using wavefield reconstruction methods, Geophysics, 81 (2016), R45-R55.

[26] J.A. Hudson, Overall properties of heterogeneous material, Geophysical Journal International, 107 (1991), 505-511.

[27] J.M. Carcione, Wavefields in real media, 3rd edition. Elsevier Science, 2014.

[28] Burridge, M.V. de Hoop, D. Miller and C. Spencer, Multiparameter inversion in anisotropic elastic media, Geophysical Journal International, 134 (1998), 757-777.

[29] H.Y. Lee, J.M. Koo, D.J. Min, B.D. Kwon and H.S. Yoo, Frequency-domain elastic full waveform inversion for VTI media, Geophysical Journal International, 183 (2010), 884-904.

[30] D. Köhn, D.D. Nil, A. Kurzmann, A. Przebindowska and T. Bohlen, On the influence of model parametrization in elastic full waveform tomography, Geophysical Journal International, 191 (2012), 325-345.

[31] M. Warner, A. Ratcliffe, T. Nangoo, J. Morgan, A. Umpleby, N. Shah, V. Vinje, I. Štekl, L. Guasch, C. Win, G. Conroy and A. Bertrand, Anisotropic 3D full-waveform inversion, Geophysics, 78 (2013), R59-R80.

[32] S. Operto, Y. Gholami, V. Prieux, A. Ribodetti, R. Brossier, L. Metivier and J. Virieux, A guided tour of multiparameter full waveform inversion with multicomponent data: From theory to practice, The Leading Edge, 32 (2013), 1040-1054.

[33] N. Kamath and I. Tsvankin, Sensitivity analysis for elastic full-waveform inversion in VTI media, 84th Annual International Meeting, SEG, Expanded Abstracts, (2014), 1162-1166.

[34] J.W. Oh and D.J. Min, Multi-parametric FWI using a new parameterization for elastic VTI media, 76th EAGE Conference and Exhibition, Amsterdam, Netherlands, 2014.

[35] T. Alkhalifah and R.-E. Plessix, A recipe for practical full-waveform inversion in anisotropic media: An analytic parameter resolution study, Geophysics, 79 (2014), R91-R101.

[36] D. Köhn, O. Hellwig, D. De Nil and W. Rabbel, Waveform inversion in triclinic anisotropic media - a resolution study, Geophysical Journal International, 201 (2015), 1642-1656.

[37] O. Podgornova, S. Leaney and L. Liang, Analysis of resolution limits of VTI anisotropy with full waveform inversion, 85th Annual International Meeting, SEG, Expanded Abstracts, (2015), 1188-1192.

[38] J.W. Oh, T. Alkhalifah and D.J. Min, Multi-stage full waveform inversion strategy for 2D elastic VTI media, 85th Annual International Meeting, SEG, Expanded Abstracts, (2015), 1204-1208.

[39] W. Pan, K.A. Innanen, G.F. Margrave, M.C. Fehler, X. Fang and J. Li, Estimation of elastic constants for HTI media using Gauss-Newton and full-Newton multiparameter fullwaveform inversion, Geophysics, 81 (2016), R275-R291.

[40] W. He and R.-E. Plessix, Analysis of different parameterizations of waveform inversion of compressional body waves in an elastic transverse isotropic earth with a vertical axis of symmetry, Geophysical Prospecting, 65 (2016), 1004-1024.

[41] J.W. Oh and T. Alkhalifah, The scattering potential of partial derivative wavefields in 3-D elastic orthorhombic media: an inversion prospective, Geophysical Journal International, 206 (2016), 1740-1760. 
[42] N. Kamath, I. Tsvankin and E. Díaz, Elastic full-waveform inversion for VTI media: A synthetic parameterization study, Geophysics, 82 (2017), C163-C174.

[43] J.W. Oh and T. Alkhalifah, Optimal full-waveform inversion strategy for marine data in azimuthally rotated elastic orthorhombic media, Geophysics, 83 (2018), R307-R320.

[44] O. Podgornova, S. Leaney and L. Liang, L.. Resolution of VTI anisotropy with elastic fullwaveform inversion: theory and basic numerical examples, Geophysical Journal International, 214 (2018), 200-218.

[45] G.E. Backus, Long-wave elastic anisotropy produced by horizontal layering, Journal of Geophysical Research, 67 (1962), 4427-4440.

[46] A. Ali and M. Jakobsen, On the accuracy of Rüger's approximation for reflection coefficients in HTI media: implications for the determination of fracture density and orientation from seismic AVAZ data, Journal of Geophysics and Engineering, 8 (2011), 372-393.

[47] M. Jakobsen and T.A. Johansen, Anisotropic approximations for mudrocks, Geophysics, 65 (2000), 1711-1725.

[48] M. Jakobsen, J.A. Hudson and T.A. Johansen, T-matrix appraoch to shale acoustics, Geophysical Journal International, 154 (2003), 533-558.

[49] M. Jakobsen and P. Rasolofosaon, Do the Velocity and Attenuation Surfaces in Anisotropic Viscoelastic Media Always Share the Same Symmetries?, 71st EAGE Conference and Exhibition incorporating SPE EUROPEC, Amsterdam, Netherlands, 2009.

[50] A. Baumstein, Extended subspace method for attenuation of crosstalk in multiparameter full wavefield inversion, 84th Annual International Meeting, SEG, Expanded Abstracts, (2014), 1121-1125.

[51] Y. Gholami, R. Brossier, S. Operto, A. Ribodetti and J. Virieux, Which parametrization for acoustic VTI full waveform inversion? Part 1: Sensitivity and tradeoff analysis, Geophysics, 78 (2013), R81-R105.

[52] X. Huang, M. Jakobsen, K. Eikrem and G. Nævdal, A target-oriented scheme for efficient inversion of time-lapse seismic waveform data, 10.1190/segam2018-2992008.1., 2018.

[53] M. Jakobsen, T-matrix approach to seismic forward modelling in the acoustic approximation, Studia Geophysica et Geodaetica, 56 (2012), 1-20.

[54] M. Jakobsen, I. Pilskog and M. Lopez, Generalized T-matrix approach to seismic modelling of fractured reservoirs and related anisotropic systems, Extended abstract, EAGE Annual Meeting, Madrid, 2015.

[55] J.R. Taylor, Scattering Theory, New York, Wiley, 1972.

[56] R.G. Newton, Scattering Theory of Waves and Particles, New York, Dover, 2002.

[57] J.E. Gubernatis, E. Domany and J.A. Krumhansl, Formal aspects of the theory of the scattering of ultrasound by flaws in elastic materials, Journal of Applied Physics, 48 (2008), 2804 (1977).

[58] V. Červený, Seismic Ray Theory, Cambridge University Press, 2001.

[59] A. Kircher and S.A. Shapiro, Fast-repeat modelling of time- lapse seismograms, Geophysical Prospecting, 49 (2001), 557-569.

[60] M. Jakobsen and J.A. Hudson, Visco-elastic waves in rock-like composites, Studia Geophysica et Geodaetica, 47 (2003), 793-826.

[61] E. Kroner, Bounds for effective moduli of disordered materials, Journal of the Mechanics and Physics of Solids, 25 (1977), 137-155.

[62] J. Willis, Mechanics of composites, Ecole polytechnique, Departement de Mecanique, 2002.

[63] V. Strassen, Gaussian Elimination is Not Optimal, Numerische Mathematik, 13 (1969), 354356. 
[64] A. Gonis and W.H. Butler, Multiple Scattering in Solids, Springer, 2000.

[65] W.C. Chew and Y.M. Wang, Reconstruction of two-dimensional permittivity distribution using distorted Born iterative method, IEEE Transactions on Medical Imaging, 9 (1990), 218225.

[66] B.A. Auld, Acoustic fields and waves in Solids, Krieger, 1990.

[67] R.C. Aster, B. Borchers and C.H. Thurber, Parameter estimation and inverse problems, Elsevier, 2013.

[68] C.G. Farquharson and D.W. Oldenburg, A comparision of automatic techniques for estimating the regularization parameter in non-linear inverse problems, Geophysical Journal International, 156 (2004), 411-425.

[69] I.R. Ciric and Y. Qin, Self-adaptive selection of the regularization parameter for electromagnetic imaging, IEEE Transactions on Magnetics, 33 (1997), 1556-1559.

[70] D. Wattzening, B. Brandsatter and G. Holler, Adaptive regularization parameter adjustment for reconstruction problems, IEEE Transactions on Magnetics, 40 (2004), 1116-1119.

[71] D. Gajewski and I. Pšenčík, Vertical seismic profile synthetics by dynamic ray tracing in laterally varying layered anisotropic structures, Journal of geophysical research, 95 (1991), 11301-11315.

[72] L. Eisner and I. Pšenčík, Computation of additional components of the first-order ray approximation in isotropic media, Pure and applied geophysics, 148 (1996), 227-253. 